\title{
Type 2 immunity in tissue repair and fibrosis
}

Richard L. Gieseck III', Mark S. Wilson ${ }^{2}$ and Thomas A. Wynn ${ }^{1}$

Abstract | Type 2 immunity is characterized by the production of IL-4, IL-5, IL-9 and IL-13, and this immune response is commonly observed in tissues during allergic inflammation or infection with helminth parasites. However, many of the key cell types associated with type 2 immune responses - including T helper 2 cells, eosinophils, mast cells, basophils, type 2 innate lymphoid cells and IL-4- and IL-13-activated macrophages - also regulate tissue repair following injury. Indeed, these cell populations engage in crucial protective activity by reducing tissue inflammation and activating important tissue-regenerative mechanisms. Nevertheless, when type 2 cytokine-mediated repair processes become chronic, over-exuberant or dysregulated, they can also contribute to the development of pathological fibrosis in many different organ systems. In this Review, we discuss the mechanisms by which type 2 immunity contributes to tissue regeneration and fibrosis following injury.

Type 2 immunity is characterized by increased production of the cytokines IL-4, IL-5, IL-9 and IL-13 (REF. 1). The $T$ helper $1\left(\mathrm{~T}_{\mathrm{H}} 1\right)$ and $\mathrm{T}_{\mathrm{H}} 2$ paradigm was first described approximately three decades $\mathrm{ago}^{2}$, and for many of the intervening years, type 2 immunity was largely considered as a simple counter-regulatory mechanism controlling type 1 immunity $^{3}$ (BOX 1). Early studies showed that when components of the type 2 response were ablated, susceptibility to a variety of autoimmune diseases generally increased due to dysregulated type 1 -driven inflammation. Today, our understanding of the diverse roles of type 2 cytokines in host immunity and inflammatory disease is becoming clearer, with type 2 immunity exhibiting either host-protective or pathogenic activity depending on the specific setting ${ }^{4}$. In addition to suppressing type 1- and $\mathrm{T}_{\mathrm{H}}$ 17-driven inflammation, type 2 immunity is directly involved in tissue repair and regeneration following injury, with many studies suggesting critical roles for IL-4- and IL-13-activated macrophages in the resolution of inflammation and restoration of tissue homeostasis $^{5,6}$. The specific mechanisms regulating tissue repair by macrophages activated by type 2 cytokines and other cell types that characterize type 2 inflammation, including eosinophils, mast cells, basophils, $\mathrm{T}_{\mathrm{H}} 2$ cells and group 2 innate lymphocytes (ILC2s), remain ill defined. Moreover, while type 2 immunity helps to restore tissue homeostasis following injury, type 2 immune responses can also lead to the development of pathological fibro$\operatorname{sis}^{7}$ (FIG. 1). The mechanisms that transform these tissueregenerative type 2 responses into progressive fibrotic disorders remain unclear, although persistent activation of tissue repair pathways is a major contributing mechanism in most cases. In this Review, we provide a brief overview of fibrotic diseases that have been linked to activation of type 2 immunity, discuss the various mechanisms that contribute to the initiation and maintenance of type 2 inflammation and examine the ways in which these pathways may become chronically activated or dysregulated. We highlight the key cell types that regulate type 2-dependent repair and fibrosis responses and illustrate both the mechanisms through which type 2 cytokines interact with other key mediators to instruct tissue repair and how these pathways contribute to the development of fibrosis when dysregulated.

\section{Diseases involving type 2 fibrosis}

Type 2 fibrosis in the liver. The liver is a key detoxifying organ that is frequently impacted by infections, injuries and insults that can lead to fibrosis over time. Chronic helminth infections evoke highly polarized type 2 immune responses that are often associated with fibroproliferative lesions, particularly during the chronic stages of infection ${ }^{4,8}$. The genus Schistosoma contains several species of trematode flatworms that currently infect over 200 million individuals worldwide? These worms lay eggs in the vasculature, and these eggs become lodged in the small venules of the liver, lung, gut and bladder, causing chronic irritation, granulomatous inflammation and fibrosis?. The fibrotic pathology that defines severe schistosomiasis has been well characterized and is largely influenced by the $\mathrm{T}_{\mathrm{H}} 2$-associated 


\section{Box 1 | Type 1 and Type 2 paradigm}

In this Review, type 1 immunity is defined by the activity of Thelper 1 cells, type 1 innate lymphoid cells, neutrophils and classically activated macrophages. Type 1 immunity is critical for defence against many intracellular pathogens, bacteria, viruses and other microorganisms. By contrast, type 2 immunity promotes immunity against extracellular parasites and helminths, helps to maintain metabolic homeostasis and regulates tissue repair following injury. Type 2 immunity is characterized by the cytokines IL-4, IL-5, IL-9, IL-13, IL-25, IL-33 and thymic stromal lymphopoietin and associated cell types, including eosinophils, mast cells, basophils, Thelper 2 cells, group 2 innate lymphoid cells and IL-4- and IL-13-activated macrophages. Many studies have shown that type 1 and type 2 immune responses display substantial cross-regulation. Therefore, therapeutic strategies that target just one arm of the immune response often lead to marked increases in the opposing response.

Alternatively activated

macrophages

These non-classically activated macrophages consist of several

functionally and transcriptionally distinct

macrophage subsets with roles in wound repair, fibrogenesis

and dampening of the

inflammatory response, among

others. These cells are

sometimes referred to as ' $M 2$ '

macrophages; however, by

convention, it is best to include

the activating stimulus in the

description, such as

'IL-4-activated macrophages'

Cholangiocarcinoma

A cancer of the bile duct epithelium that is relatively

rare in the Western world but more common in areas

endemic for liver flukes.

Primary sclerosing cholangitis

An idiopathic disorder resulting in chronic inflammation of the bile ducts that progresses to ductal narrowing and eventual ductal loss, followed by replacement with fibrotic tissue.

\section{Biliary atresia}

A disease characterized by the progressive loss of bile ducts

usually in early childhood. Although the cause of biliary atresia is debated, there is evidence to suggest an autoimmune component to the disease that is precipitated by infection or the passage of maternal toxins across the placental barrier. cytokines IL-4, IL-5 and IL-13 and associated accessory cells, including eosinophils and IL-4- and IL-13-induced alternatively activated macrophages ${ }^{10,11}$.

Closely related are liver flukes, including the species Clonorchis sinensis and Opisthorchis viverrini, which currently affect over 50 million individuals worldwide owing to consumption of undercooked freshwater fish $^{12}$. These worms, which reside in the large branching bile ducts, feed both on the cholangiocytes lining the bile ducts and on bile itself, causing extreme biliary hyperplasia that often progresses to cholangiocarcinoma ${ }^{13,14}$. Liver flukes induce IL-4, IL-5 and IL-13 responses ${ }^{15-17}$, and it has been suggested that the fibrosis associated with liver fluke infection is IL-13-dependent ${ }^{16,17}$.

In addition to parasites, the development of type 2 cytokine-driven fibrosis has been linked to several other pathogens and hereditary diseases. Subsets of patients with hepatitis $\mathrm{C}$ virus infection, primary sclerosing cholangitis, primary biliary cirrhosis, autoimmune hepatitis, biliary atresia and non-alcoholic steatohepatitis (NASH) exhibit significantly increased levels of IL-4, IL-5 and/or IL-13 in addition to many type 1 - and $\mathrm{T}_{\mathrm{H}} 17$ cell-associated cytokines ${ }^{18,19}$. However, the specific contributions of these cytokines in these diseases remain less clear.

Type 2 fibrosis in the lung. Like the liver, which has the capacity to regenerate from as little as $25 \%$ of its original mass ${ }^{20}$, the lung is remarkably resilient following injury and exhibits substantial reparative ability ${ }^{21}$. Nevertheless, in response to chronic injury and insults, the wound healing pathways can become overactive and dysregulated, resulting in poor regenerative potential along with increasing fibrosis. For these reasons, pulmonary diseases, including asthma, chronic obstructive pulmonary disease (COPD) and idiopathic pulmonary fibrosis (IPF), are very common. These diseases affect hundreds of millions of people globally and represent some of the leading causes of chronic morbidity and mortality ${ }^{22,23}$. Restoration of the normal architecture of the lung following injury requires a rapid and controlled repair process, which, if uncoordinated, can result in local scarring, with fibrotic lesions forming in what is otherwise a highly elastic and flexible organ. This dysregulated repair of pulmonary tissue following infection or insults from environmental triggers can predispose individuals to subsequent infections ${ }^{24}$ and cause lung dysfunction ${ }^{25}$. Thus, pulmonary fibrosis is of significant medical concern, and there are very few effective treatment options. Although it is difficult to distinguish cause from consequence, pulmonary fibrosis has been observed in an array of pulmonary diseases, including acute respiratory distress syndrome ${ }^{26}$, asbestosis $^{27}$, asthma ${ }^{28}$, bacterial infection ${ }^{29}$, bronchiolitis ${ }^{30}$, $\mathrm{COPD}^{31}$, cystic fibrosis ${ }^{32}$, emphysema ${ }^{33}$, lung cancer $^{34}$, pneumonia $^{35}$, IPF and sarcoidosis ${ }^{36}$.

Whether common mechanistic pathways among these diseases contribute to dysregulated wound healing and fibrotic scaring is not clear. Two prevailing upstream cytokine-driven inflammatory pathways in the lung have been identified, involving transforming growth factor $\beta$ (TGF $\beta$ ) and/or IL-13 (REF. 37). Several years ago, we and others identified that activation of an IL-1-IL-17A-associated axis can propagate a TGF $\beta$-dependent pulmonary fibrogenic pathway ${ }^{38-40}$. Although elevated levels of IL-17A and excessive activation of IL-17A-dependent pathways contribute to the inflammatory milieu in many of the above-mentioned pulmonary diseases ${ }^{41-47}$, it is currently unclear whether IL-17A directly contributes to the development of pulmonary fibrosis. Type 2 cytokines, and specifically IL-4 and IL-13, are elevated in many of these pulmonary diseases ${ }^{48-57}$, with IL-13 attracting substantial attention as a therapeutic target for asthma ${ }^{58}$. Promising data from preclinical studies led to the development of several clinical trials targeting IL-13. In particular, two humanized anti-IL-13 monoclonal antibodies (tralokinumab ${ }^{59}$ and lebrikizumab ${ }^{60}$ ) and an anti-IL-4Ra monoclonal antibody (dupilumab ${ }^{61}$ ) have been tested in patients with mild-to-moderate or severe asthma and were found to reduce the frequency of asthma exacerbations and to improve lung function in some, but not all, studies. For example, despite encouraging preclinical data, tralokinumab recently failed to meet its primary end point of reducing the annual exacerbation rate in the first of two phase III trials in severe uncontrolled asthma. Although lebrikizumab showed mixed results in asthma, clinical studies in COPD, atopic dermatitis and IPF are ongoing. Unfortunately, airway remodelling and fibrosis severity were not reported in the asthma trials. Increased innate and adaptive immune cellderived IL-13 and elevated expression of IL-13Ra1 and IL-13R 22 have been observed in patients with IPF ${ }^{62-65}$, further supporting the hypothesis that IL-13 contributes to pulmonary fibrosis. Indeed, using a humanized severe combined immunodeficiency (SCID) mouse model of IPF, in which mice are infused with fibroblasts from patients with IPF, Murray and colleagues observed a significant reduction in fibrosis and increased repair of the airway epithelium following anti-IL-13 (tralokinumab) treatment ${ }^{66}$. In relation to IL-13-driven pulmonary fibrosis, both TGF $\beta$-dependent ${ }^{67,68}$ and TGF $\beta$-independent ${ }^{37}$ mechanisms have been proposed; however, it remains unclear whether therapeutically targeting IL-13 or any of the TGF $\beta$ family members would provide a clinical benefit in fibroproliferative diseases of the lung and to what degree IL-17A and TGF $\beta$ interact with type 2 immunity in various aetiologies of pulmonary fibrosis. 


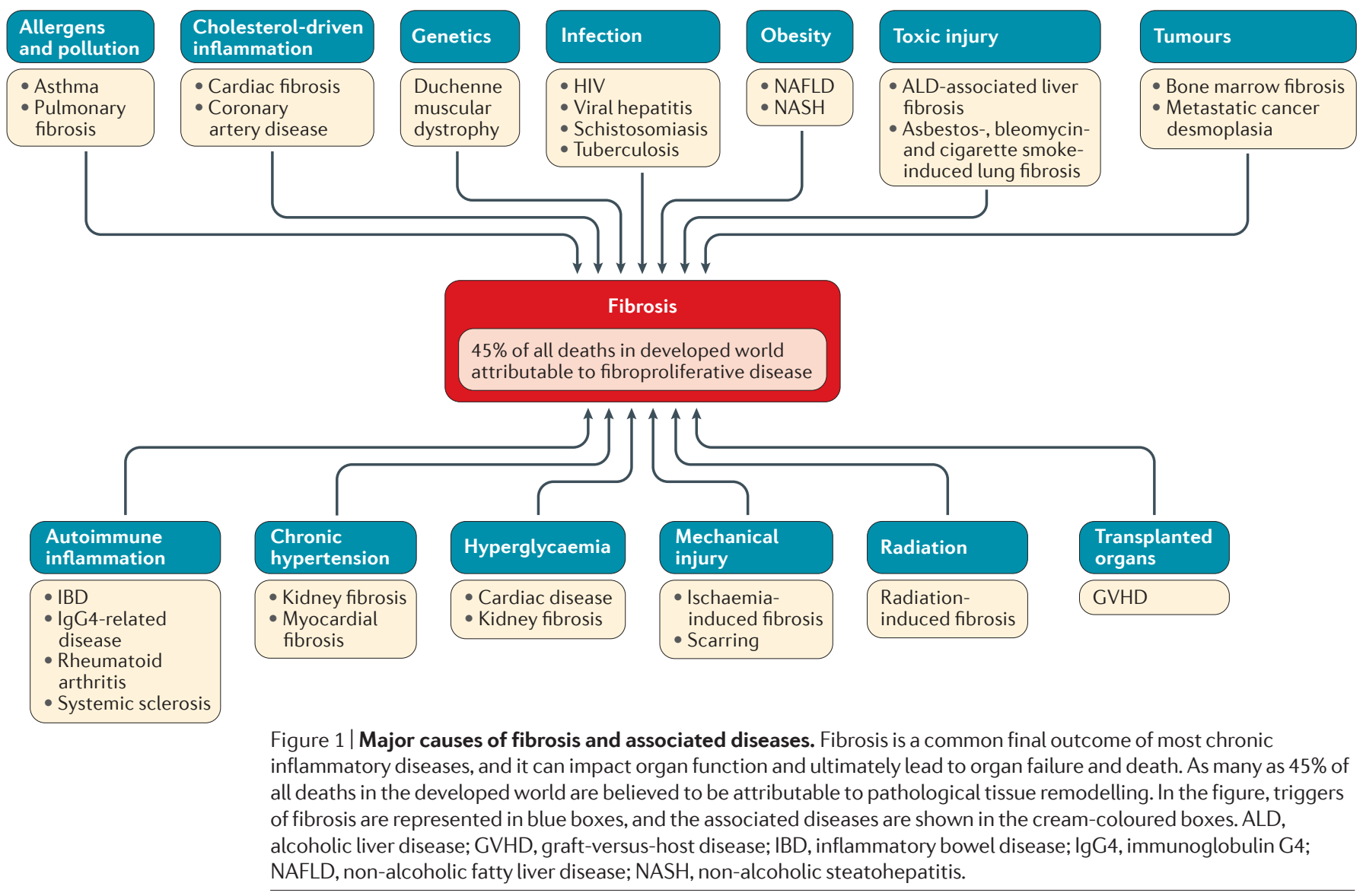

Type 2 fibrosis in other organs. Type 2 fibrosis can affect nearly every organ in the body; however, due to space limitations, we will focus mainly on examples from the liver and lung. Nevertheless, there are a few other noteworthy examples that will be highlighted. IgG4-related disease is a chronic inflammatory disease characterized by high levels of infiltrating IgG4-secreting plasma cells. This disease has distinct manifestations that can affect disparate organs; however, several commonalities have emerged over the past few years, including infiltration of eosinophils and progression to fibrosis ${ }^{69}$, perhaps suggesting a role for type 2 immunity in disease pathogenesis. The anti-CD20 antibody rituximab, which depletes B cells, has shown some efficacy in patients with IgG4-related disease $^{70}$; nevertheless, additional mechanistic work is needed to determine the role of B cells and IgG4 relative to other cell types, including $\mathrm{T}_{\mathrm{H}} 2$ cells and eosinophils, and whether they contribute to the development of fibrosis in this disease.

Atopic dermatitis is a disease in which the accumulation of mast cells and eosinophils within the skin drives chronic pruritus and thickening of the skin, resulting in a significant loss of quality of life for patients ${ }^{71}$. Recent studies have identified the alarmins IL-25 and IL-33 as well as ILC2s as key drivers of atopic dermatitis $^{72,73}$. Furthermore, in clinical trials using dupilumab, a humanized monoclonal antibody that blocks both IL-4 and IL-13 signalling, $85 \%$ of patients receiving the antibody met the primary end point of a $50 \%$ decrease in the eczema area and severity score, compared with only $35 \%$ of patients in the placebo $\mathrm{arm}^{74}$.

Another noteworthy disease, ulcerative colitis, is a chronic inflammatory bowel disease that results in severe epithelial damage and ulceration of the large intestine $\mathrm{e}^{75}$. The cytokine response observed in ulcerative colitis is complex ${ }^{76}$; however, elevation of type 2 cytokines has been noted in the lamina propria of the gut in animal models of ulcerative colitis and in patients with active disease, suggesting that blockade of these cytokines may be efficacious ${ }^{77,78}$. Nevertheless, two clinical trials in which IL-13 was blocked (using tralokinumab $^{79}$ or anrukinzumab ${ }^{80}$ ) in ulcerative colitis failed to meet their primary end points. Surprisingly, patients receiving higher doses of anrukinzumab fared worse than those receiving lower doses ${ }^{80}$. It is interesting to speculate that blocking type 2 immunity in diseases with mixed cytokine responses, such as ulcerative colitis, may dysregulate important regulatory pathways that keep type 1 - and $\mathrm{T}_{\mathrm{H}} 17$-driven inflammation in check. Crossregulation between type 1 and type 2 immunity, particularly during therapeutic interventions, is a key focus of this Review and will be discussed in greater detail below.

\section{Initiation of type 2-driven fibrosis}

Role of alarmins in the type 2 response and fibrosis. Mucosal epithelial cells of the airways and intestine both provide physical barriers to the environment and possess 


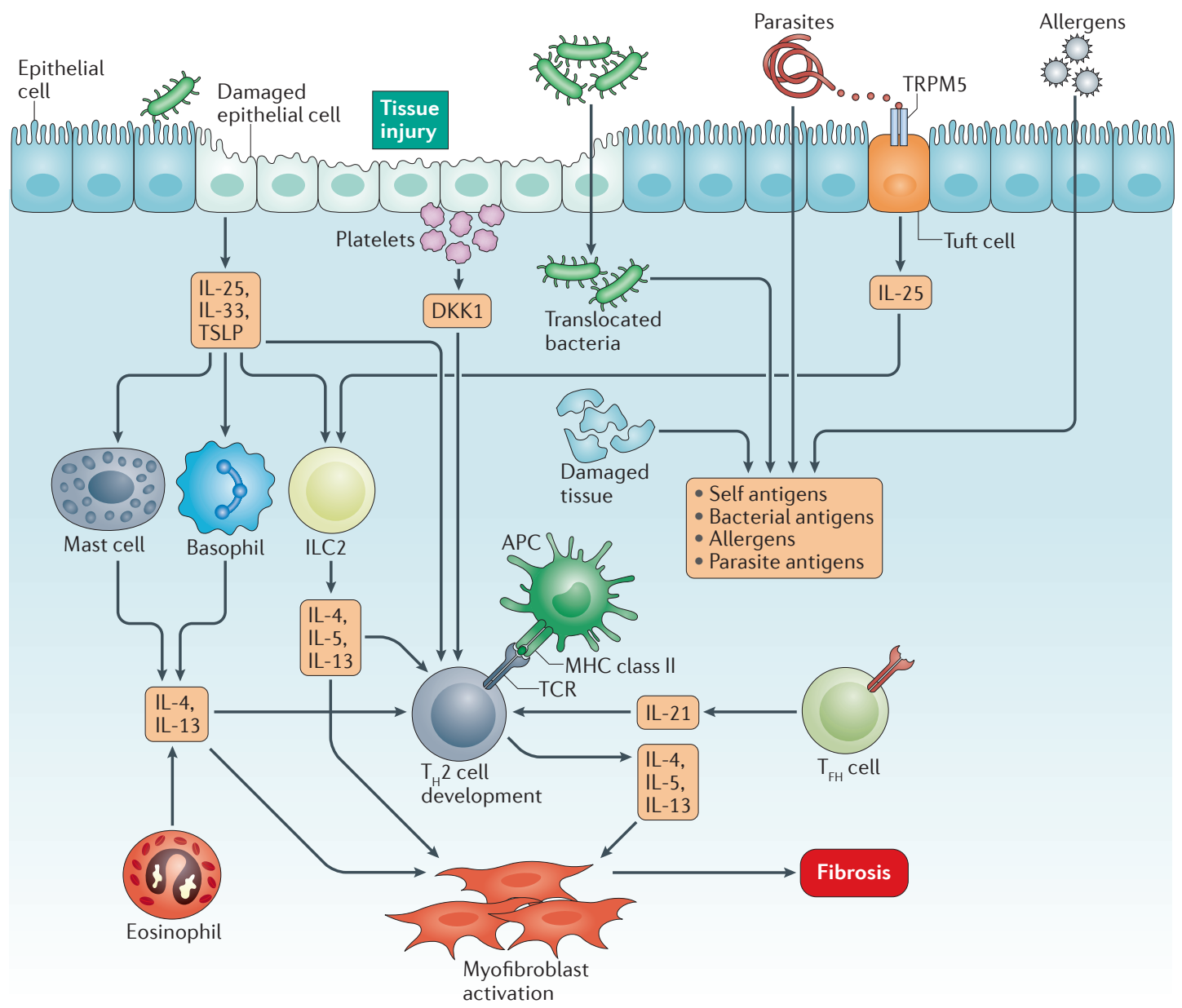

Figure 2 | Initiation of type 2 immune responses. Following tissue injury, damaged epithelial cells secrete IL-25, IL-33 and thymic stromal lymphopoietin (TSLP). These alarmins signal through innate cells, including basophils, mast cells and group 2 innate lymphoid cells (ILC2s), resulting in the production of the type 2 cytokines IL-4, IL-5 and IL-13. These cytokines support naive $C D 4^{+} T$ cell differentiation into $T$ helper $2\left(T_{H} 2\right)$ cells and support $T_{H} 2$ cell effector functions. Dickkopf-related protein 1 (DKK1) secreted from platelets at the site of injury can similarly support the differentiation of $\mathrm{T}_{\mathrm{H}} 2$ cells. Bacterial translocation and self-antigens that are exposed at the site of tissue injury as well as antigens from allergens and parasites can be presented to T cells by antigen-presenting cells (APCs). Additionally, parasite antigens can

Tuft cells

A very rare chemo-sensing epithelial cell type that is spread throughout the gastrointestinal and respiratory tracts. Tuft cells secrete the alarmin IL-25 in response to parasitic infections and perhaps other stimuli and are thought to be important sentinels for and innate initiators of the type 2 response.

\section{Goblet cells}

A specialized, mucus-secreting epithelial cell found throughout the gastrointestinal and respiratory tracts and across mucosal surfaces. Goblet cells and their secreted mucins are critical for protecting mucosal surfaces, maintaining barrier integrity and removing large extracellular irritants and debris.

be detected by specialized epithelial cells, known as tuft cells, through chemo-sensing receptors such as transient receptor potential cation channel subfamily M member 5 (TRPM5) and gustducin (not depicted), resulting in IL-25 release, which subsequently activates ILC2s. IL-21 produced by follicular helper $T\left(T_{F H}\right)$ cells and IL-4 and IL-13 released by eosinophils can augment these processes. IL-4 and IL-13 secreted from basophils, mast cells, eosinophils and ILC2s also directly contribute to the activation of myofibroblasts and the development of fibrosis. TCR, T cell receptor.

instructive properties to guide immune responses s1,82 $^{8}$. Recent studies have identified a variety of epithelial cells, including IL-25-secreting tuft cells ${ }^{83-85}$, antigen-sensing goblet cells ${ }^{86}$ and phagocytic epithelial cells ${ }^{87}$, suggesting a greater division of labour among the specialized cells of the mucosal epithelium than previously appreciated. Originally identified as a T cell-derived cytokine ${ }^{88,89}$, IL-25 can initiate a type 2 cytokine cascade involving the induction of IL-4, IL- 5 and IL-13. Several years after the initial identification of IL-25, epithelial expression of ACT1 (which encodes TRAF3 interacting protein 2 and is also known as TRAF3IP2) was shown to potentiate IL-25 signalling through a non-lymphocyte, nonnatural killer (NK) cell, non-granulocyte lineage, which we now know as ILC2 $s^{90}$. Around the same time, it was observed that epithelial cell-derived thymic stromal lymphopoietin (TSLP) activated local dendritic cells (DCs) to secrete CC chemokine ligand 22 (CCL22) and CCL17, promoting $\mathrm{T}_{\mathrm{H}} 2$ cell differentiation and propagating type 2 allergic inflammation. A third member of the alarmins, IL-33, was also identified as a $\mathrm{T}_{\mathrm{H}} 2$ cell-promoting cytokine ${ }^{91}$ that signals through IL-1 receptor-like 1 (IL1RL1; also known as protein ST2) on mast cells and $\mathrm{T}_{\mathrm{H}} 2$ cells; however, it has since been shown that IL-33 also has important $\mathrm{T}$ cell-independent immune functions ${ }^{92-94}$.

Alarmins are considered to be some of the earliest of the type 2 cytokines to be secreted following tissue damage, and they have the capacity to both initiate and propagate a fulminant type 2 immune response (FIG. 2). If left unchecked, the alarmins can also contribute to the initiation and progression of fibrosis, with indirect 
actions via innate or adaptive immune cells. For example, both IL-13-secreting ILC2s and IL-25 have been observed in patients with IPF, leading Hams and colleagues ${ }^{95}$ to examine the role of IL-25 in the development of IPF. Indeed, using Schistosoma mansoni eggs, they showed that IL-25-activated ILC2s exhibited substantial production of the pro-fibrotic cytokine IL-13, indicating that this innate axis may contribute to human pulmonary fibrosis. Similarly, intranasal delivery of recombinant IL-25 in mice led to airway inflammation, connective tissue growth factor (CTGF) and TGF $\beta 1$ production and pulmonary fibrosis ${ }^{96}$. Similar observations have been made for IL-33, with elevated levels of IL-33 detected in dermal tissues during cutaneous fibrosis ${ }^{97,98}$, in the lung or intestinal epithelium of patients with pulmonary fibrosis or fibrotic colitis ${ }^{99-102}$ and in the liver of mice with hepatic fibrosis ${ }^{99}$. Mechanistically, it has been proposed that IL-33 drives fibrosis by inducing IL-13 production by ILC2s, macrophages ${ }^{103}$ and eosinophils ${ }^{100}$. TSLP has also been observed in human and experimental pulmonary fibrosis ${ }^{104,105}$, systemic sclerosis ${ }^{106-108}$, colonic fibrosis ${ }^{109}$ and skin fibrosis ${ }^{110}$. Gain-of-function and loss-of-function experiments indicate that TSLP has pro-fibrotic properties, triggering mitogen-activated protein kinase signalling pathways in fibroblasts, which leads to the synthesis of collagens ${ }^{111-113}$. Disruption of TSLP receptor signalling also reduced IL-13-driven ${ }^{110}$ and TGF $\beta$-driven ${ }^{108}$ fibrosis, suggesting that TSLP can also operate downstream of IL-13 and TGF $\beta$; however, these results need to be verified. Additionally, there is some evidence suggesting that epithelial-derived IL-1a may be an upstream initiator of IL-33 and TSLP and that disruption of IL-1 $\alpha$ signalling may prevent allergic priming by preventing DC migration ${ }^{14}$.

Given the overlapping expression and the possible context-dependent function of the alarmins, we investigated the potential redundancy between the alarmins by inhibiting IL-25, IL-33 and TSLP individually or collectively following the initiation of allergen-induced airway inflammation, chronic S. mansoni-driven hepatic fibrosis or acute pulmonary fibrosis in mice ${ }^{115}$. Blockade of IL-25, IL-33 or TSLP had no impact on IL-13-mediated chronic hepatic or acute pulmonary fibrosis; however, blockade of all three alarmins significantly reduced inflammation, ILC2 recruitment, eosinophilia and fibrosis ${ }^{115}$. During chronic house dust mite (HDM)-driven airway inflammation, ablation of all three alarmins had no impact on established disease; however, blockade during the priming phase prevented the establishment of inflammation and the progression of airway pathology, suggesting that combined therapeutic intervention may be required to temper type 2-driven fibrosis in some settings.

Role of adaptive $T_{H} 2$ cell responses in fibrosis. The activation of innate immune cells, including basophils ${ }^{116}$ and ILC2 $\mathrm{s}^{117}$, contributes to the local and early secretion of IL-4, IL-5 and IL-13. Following T cell receptor (TCR) engagement and co-stimulation, IL-4 receptor signalling via signal transducer and activator of transcription 6 (STAT6) and GATA3 in T cells instructs a $\mathrm{T}_{\mathrm{H}} 2$ cell differentiation programme ${ }^{118}$ (FIG. 2). More than 20 years ago, Cheever and colleagues identified an important role for IL- 4 and $\mathrm{T}_{\mathrm{H}} 2$ cells in the development of S. mansoni- and S. japonicum-associated hepatic fibro$\operatorname{sis}^{119,120}$. In these cases, IL-13 was continuously secreted by $\mathrm{T}_{\mathrm{H}} 2$ cells specific for parasite antigen, and this orchestrated a fibrotic response around tissue-trapped parasite eggs ${ }^{121}$. Similarly, fungal- and viral-associated pulmonary fibrosis ${ }^{122,123}$, in addition to post-irradiation ${ }^{124}$ and silica-induced pulmonary fibrosis, have been associated with the accumulation of $\mathrm{T}_{\mathrm{H}} 2$ cells. $\mathrm{T}_{\mathrm{H}} 2$ cell-associated fibrosis is not restricted to the lung and liver; experimental renal fibrosis ${ }^{125,126}$, soft-tissue fibrosis ${ }^{127}$ and peritoneal fibrosis ${ }^{128}$ have also been associated with the accumulation of $\mathrm{T}_{\mathrm{H}} 2$ cells.

The antigen specificity of $\mathrm{T}_{\mathrm{H}} 2$ cells in many of these settings is unclear. Bacteria-specific $\mathrm{T}_{\mathrm{H}} 2$ cells have been reported ${ }^{129,130}$; however, it is unclear whether local microorganisms contribute to $\mathrm{T}_{\mathrm{H}} 2$ cell activation and accumulation at fibrotic sites. It has also been suggested that self-antigens that are exposed following local tissue damage could promote the activation of $\mathrm{T}_{\mathrm{H}} 2$ cells at damaged tissue sites ${ }^{131}$; however, this needs to be clarified. Alternatively, recent studies have shown that $\mathrm{T}_{\mathrm{H}} 2$ cells can produce IL-13 in direct response to alarmins, allowing these cells to adopt innate-like properties in response to tissue-derived cytokines ${ }^{132-134}$.

In addition to IL-4, a recent study identified plateletderived Dickkopf-related protein 1 (DKK1), a Wnt antagonist, as an important contributor to $\mathrm{T}_{\mathrm{H}} 2$ cell differentiation in vitro in combination with TCR engagement and during $\mathrm{T}_{\mathrm{H}} 2$ cell-mediated airway allergy and immunity ${ }^{135}$. This study provides physiological and mechanistic links among local tissue damage, platelet activation and $\mathrm{T}_{\mathrm{H}} 2$ cell differentiation in addition to the well-established role of platelets as a key source of platelet-derived growth factor (PDGF). Whether additional tissue-derived signals contribute to $\mathrm{T}_{\mathrm{H}} 2$ cell differentiation following local tissue damage is unclear. More detailed experimental analysis of the TCR repertoire of $\mathrm{T}_{\mathrm{H}} 2$ cells at fibrotic sites is required to determine the antigen specificity of $\mathrm{T}_{\mathrm{H}} 2$ cells and potential pathogenic stimuli. Nevertheless, as they have the capacity to secrete both inflammatory- and regulatory-type cytokines and chemokines ${ }^{136}, \mathrm{~T}_{\mathrm{H}} 2$ cells can recruit and activate innate and adaptive immune cells that are involved in both initiating and resolving inflammation.

It has long been appreciated that $\mathrm{T}_{\mathrm{H}} 2$ cell-derived IL-4 and IL-13 can alternatively activate macrophages ${ }^{137}$. We showed that a follicular helper $\mathrm{T}\left(\mathrm{T}_{\mathrm{FH}}\right)$ cell-associated cytokine, IL-21, could accentuate the effects of IL- 4 and IL-13 on macrophages by upregulating their expression of IL-4Ra and IL-13Ra 1 (REF. 138). IL-21R-deficient mice showed a significant reduction in $\mathrm{T}_{\mathrm{H}} 2$ cell-mediated fibrosis and immunity following S. mansoni or Nippostrongylus brasiliensis infection ${ }^{138}$ supporting a critical role for IL-21 in amplifying $\mathrm{T}_{\mathrm{H}} 2$ cell-mediated pathologies. These observations were supported by Coquet and colleagues ${ }^{139}$, who identified allergen-specific, tissue-associated IL-21-secreting $\mathrm{T}$ cells that were distinct from $\mathrm{T}_{\mathrm{H}} 2$, $\mathrm{T}_{\mathrm{H}} 17$ and $\mathrm{T}_{\mathrm{FH}}$ cells. Similar to our observations, these 
authors found that IL-21R-deficient mice developed reduced allergen-driven airway inflammation. In addition to the inflammatory role of $\mathrm{T}_{\mathrm{H}} 2$ cells, $\mathrm{T}_{\mathrm{H}} 2$ cell-derived cytokines can stimulate local fibroblasts, endothelial cells and epithelial cells, extending their influence beyond the immune system ${ }^{140}$, as we describe in more detail below.

Role of metabolism in type 2 responses. Local and systemic energy resources influence all physiological responses, including normal organ function, tissue regeneration and repair. Close relationships among metabolism, adipose tissue dysfunction, type 2 immunity and fibrosis ${ }^{141}$ are slowly being revealed. The immune system is particularly sensitive to metabolite availability, and several recent studies have highlighted a two-way interaction between type 2 immune responses and metabolic programmes. For example, the differentiation of $\mathrm{T}_{\mathrm{H}} 2$ cells from quiescent naive $\mathrm{T}$ cells requires mammalian target of rapamycin complex 1 (mTORC1)dependent and regulatory-associated protein of mTOR (RAPTOR)-dependent metabolic reprogramming, which increases glycolysis and glucose metabolism ${ }^{142}$. By contrast, alternative activation of macrophages by IL-4 and IL-13 requires tumour progression locus 2-dependent lipid oxidative metabolism ${ }^{143,144}$ to both regulate $\mathrm{T}_{\mathrm{H}} 2$ cell proliferation and limit fibrotic responses ${ }^{144,145}$. In return, type 2 immune responses also influence adipocyte metabolism, providing a feedback loop between metabolic and immune responses. Specifically, ILC2-derived IL-5 and IL-13 and eosinophil-derived IL-4 regulate adipocyte development, beige adipogenesis and caloric expenditure ${ }^{143,146-148}$. The interactions between type 2 immunity and metabolism $^{149}$ and the metabolic requirements of fibrosis ${ }^{150-152}$ have been extensively reviewed elsewhere ${ }^{153}$. However, it is interesting to speculate that targeting the metabolic requirements of type 2 immunity may starve type 2 immune responses and slow the progression of type 2-dependent fibrosis ${ }^{154}$.

\section{Cell-specific roles in type 2 fibrosis}

Role of monocytes and macrophages. Monocytes and macrophages are crucial regulators of the initiation, maintenance and resolution of repair following injury and dictate whether wounds regenerate successfully or progress to pathological fibrosis. To achieve these disparate functions, monocytes and macrophages undergo major phenotypic, functional and metabolic changes in response to signals found in the local milieu, which result in substantial functional changes in the surrounding cells and extracellular matrix ${ }^{7,155,156}$. These factors prove critical in determining whether the microenvironment favours ongoing inflammation, regeneration and/or fibrosis following injury. However, elucidation of the specific roles of monocytes and macrophages has proven challenging, in part due to the tremendous plasticity and functional diversity of these cells, which are influenced by numerous factors, including the origin of the cells, the site of injury, the phase of injury and the aetiology of the disease ${ }^{7}$.
For example, recent work using CD11b-diphtheria toxin receptor transgenic mice, with deletion of CD11b-expressing cells, demonstrated that $\mathrm{CD}_{11 \mathrm{~b}}{ }^{+} \mathrm{F} 4 / 80^{+} \mathrm{LY}_{6 \mathrm{C}^{+}}$inflammatory macrophages are necessary to maintain fibrosis through the continued recruitment and activation of effector $\mathrm{T}_{\mathrm{H}} 2$ cells within the lung and that depletion of macrophages reduced fibrosis and inflammation by reducing the number of $\mathrm{T}_{\mathrm{H}} 2$ cells within the tissue ${ }^{157}$. By contrast, studies investigating the specific contribution of tissue-resident IL-4and IL-13-activated macrophages suggested that these cells were anti-fibrotic, as mice with a tissue macrophage-specific deletion of IL-4Ra displayed increased inflammation but little change in hepatic fibrosis following infection with $S$. mansoni. The authors concluded that IL-4R $\alpha$-expressing macrophages may reduce inflammation and fibrosis by competing with $\mathrm{T}$ cells and possibly myofibroblasts for essential metabolites like L-arginine ${ }^{145,158}$. However, it is important to note that these studies were carried out in the liver, and subsequent studies investigating this phenomenon in the asthmatic lung failed to find a pathogenic or protective role for arginase-expressing myeloid cells, suggesting organ- or aetiology-specific differences in the role of these cells ${ }^{159}$.

Macrophage and monocyte ontogeny can also affect the role of these cells in regulating disease. For example, a novel population of atypical monocytes derived from granulocyte and macrophage progenitors was recently reported to be critical for bleomycin-induced lung fibrosis. These atypical CEACAM1 ${ }^{+} \mathrm{MSR} 1^{+} \mathrm{LY}_{6 \mathrm{C}^{-}}$ $\mathrm{F} 4 / 80^{-} \mathrm{CD} 11 \mathrm{~b}^{+}$cells, which were termed 'segregatednucleus-containing atypical monocytes', were a critical source of tumour necrosis factor (TNF), and their depletion prevented bleomycin-induced fibrosis ${ }^{160}$. Studies using $\mathrm{Il}_{4 \mathrm{ra}}{ }^{-/ \text {Flox }} \mathrm{Lyz}^{\text {cre }}$ transgenic mice, in which IL-4Ra is deleted in tissue-resident macrophages (which are LysM $^{\mathrm{hi}}$ ), but not in newly recruited monocytes (which are Lys $\mathrm{M}^{\text {low }}$ ), revealed an important difference between recruited and tissue-resident monocytes and macrophages in regulating the pathogenesis of S. mansoniinduced fibrosis. IL- $4 \mathrm{Ra}^{+}$tissue-resident macrophages suppressed inflammation in the tissue, whereas recruited LysM $^{\text {low }}$ monocytes were shown to slow the progression of type 2 cytokine-driven fibrosis ${ }^{161}$.

Monocytes and macrophages can also directly influence fibrogenesis through substrate and matrix interactions. During macrophage polarization, the way in which macrophages utilize L-arginine changes drastically. Interferon $\gamma$ (IFN $\gamma)$-activated macrophages activate inducible nitric oxide synthase to produce nitric oxide and citrulline, whereas IL-4- and IL-13-activated macrophages utilize arginase to produce ornithine, polyamines and proline, the last of which is a critical building block of collagen (as reviewed previously ${ }^{162}$ ). Thus, competition for L-arginine can directly influence the progression of fibrosis by limiting the substrates available for collagen synthesis. Conversely, macrophagesecreted matrix metalloproteinases (MMPs) regulate the progression and resolution of fibrosis by controlling matrix degradation and by regulating local inflammation 
via facilitation of both extravasation and recruitment of inflammatory cells ${ }^{163}$. MMP12 is produced by IL-4- and IL-13-activated macrophages and can suppress the expression of the collagenolytic proteins MMP2, MMP9 and MMP13, resulting in diminished matrix degradation and augmented fibrotic responses following infection ${ }^{164}$. It has also been noted that macrophages are a key source of factors that regulate fibroblast growth, differentiation and survival, such as TGF $\beta$, PDGF and several members of the fibroblast growth factor family ${ }^{7,155,156}$. During skin repair, resistin-like a (RELM $\alpha$ ) secreted from IL-4- and IL-13-activated macrophages activates fibroblasts, which upregulate lysyl hydroxylase 2, a protein that regulates the mechanical crosslinking of collagen fibrils ${ }^{165}$. Other studies have shown that RELMa secreted from IL-4and IL-13-activated macrophages is also critical in modulating type 2 cytokine production ${ }^{166,167}$.

Finally, two recent studies have suggested that IL-4 and IL-13 may not be sufficient for the activation of tissue repair macrophages and that engagement of local tissue signals is also required ${ }^{168,169}$. Additionally, several recent studies have identified that IL-33 signalling may be important for the developmental remodelling by IL-4- and IL-13-activated macrophages that occurs in adipose tissues and the lung ${ }^{170,171}$. It is interesting to speculate that age-, tissue- and injury-specific co-signals may account for some of the differences in macrophage function observed across different organs and various aetiologies of disease. Together, these examples illustrate the diverse and multifaceted functional roles of monocytes and macrophages in both driving and regulating inflammation, tissue repair and fibrosis (FIG. 3).

Role of eosinophils in type 2 fibrosis. Eosinophils are considered to be potent drivers of inflammatory damage, and they secrete type 2 cytokines and TGF $\beta 1$ (REFS 172,173) in addition to many highly reactive enzyme-containing granules. Indeed, early studies in Il5 ${ }^{-/-}$mice suggested a prominent role for eosinophils in the development of type 2 fibrosis in the liver ${ }^{174}$. In these experiments, $I l 5^{-1-}$ mice infected with S. mansoni had a $40 \%$ reduction in fibrosis, and this was associated with reduced levels of IL-13. As expected, liver granulomas were almost completely devoid of eosinophils. However, it is important to note that these mice had developmental defects, including a lack of $\mathrm{CD}^{+} \mathrm{B}$ cells, that may have contributed to the observed phenotype ${ }^{175}$. Subsequent studies using $\triangle$ dblGATA mice and TgPHIL mice, which are also deficient in eosinophils, found that these mice display little to no defect in type 2 cytokine production, granulomatous inflammation or fibrosis following infection, thus calling into question the overall importance of eosinophils in fibrosis ${ }^{176}$. Nevertheless, therapeutic anti-IL-5 antibodies, such as mepolizumab, have demonstrated moderate success in ameliorating disease in human hypereosinophilic syndromes as well as in eosinophilic asthma ${ }^{177,178}$. Studies have also suggested that IL-5 may have an important role in type 2 fibrosis beyond the simple activation and recruitment of eosinophils. Still, other studies have demonstrated that the role of eosinophils in some fibrotic disorders can be quickly compensated for by other cells, including ILC2s, basophils, mast cells, NK cells and natural killer T cells.

Eosinophils have also been linked to the development of lung and skin fibrosis in chronic asthma and atopic dermatitis as well as vascular injury following high-dose irradiation, with various eosinophil granule proteins and pro-fibrotic cytokines playing key roles ${ }^{28,179-182}$. Distinct tissue-resident and recruited inflammatory eosinophil subsets with unique functional roles in $\mathrm{T}_{\mathrm{H}} 2$ cell-driven allergic inflammation were also recently described ${ }^{183}$. Consequently, it is interesting to speculate that the recruited eosinophil subset could be the key inflammatory population driving the development of $\mathrm{T}_{\mathrm{H}} 2$ cell-dependent fibrosis. Alternatively, decreases in the regulatory tissue-resident eosinophil population could augment type 2-mediated inflammation and exacerbate pulmonary fibrosis in the lung ${ }^{183}$.

We recently showed elevated expression of the type 2 cytokines IL-4, IL-5 and IL-13 in murine models of non-alcoholic fatty liver disease (NAFLD) and additionally observed that serum levels of IL-4, IL-5 and IL-13 are highly correlated with METAVIR fibrosis scores in human patients with NAFLD or NASH, suggesting a role for type 2 immunity in the progression of NASHassociated liver fibrosis ${ }^{184}$, which is quickly becoming the number-one indication for liver transplantation in the developed world. The presence of eosinophil-related gene signatures in liver biopsy samples could be used to distinguish patients with benign steatosis from those with inflammatory NASH. Additionally, eosinophils were observed near steatotic and fibrotic regions in both mice and humans; however, more work is needed to clarify whether these cells contribute to the pathogenesis of liver fibrosis.

To this point, eosinophils were also recently linked to tissue regeneration following injury to the liver or skeletal muscle. Indeed, a recent study found that eosinophils regulated liver regeneration following carbon tetrachloride intoxication and two-thirds partial hepatectomy by secreting IL- 4 and IL-13, which act as hepatocyte mitogens in an IL-4Ra-dependent manner ${ }^{185}$. Similar findings were also reported in the case of skeletal muscle injury. However, in this case, eosinophil-derived IL-4 targeted the regenerative functions of muscle-resident fibrocyte and adipocyte progenitor cells that support myogenesis ${ }^{186}$. Thus, while the function of eosinophils in pathogenic fibrosis is somewhat variable, these cells clearly play important roles in tissue homeostasis following injury and may preferentially promote tissue regeneration in some settings (FIG. 3).

Role of fibroblasts in type 2 fibrosis. In contrast to the complex roles of many of the cell types discussed thus far, fibroblasts inarguably play a critical role in normal wound healing and the pathogenesis of fibrosis through the direct deposition of extracellular matrix in response to a diverse set of cytokines and growth factors (as recently reviewed ${ }^{187}$ ). Fibroblasts also serve as a vital source of cytokines, growth factors and chemotactic factors that influence the local microenvironment and 


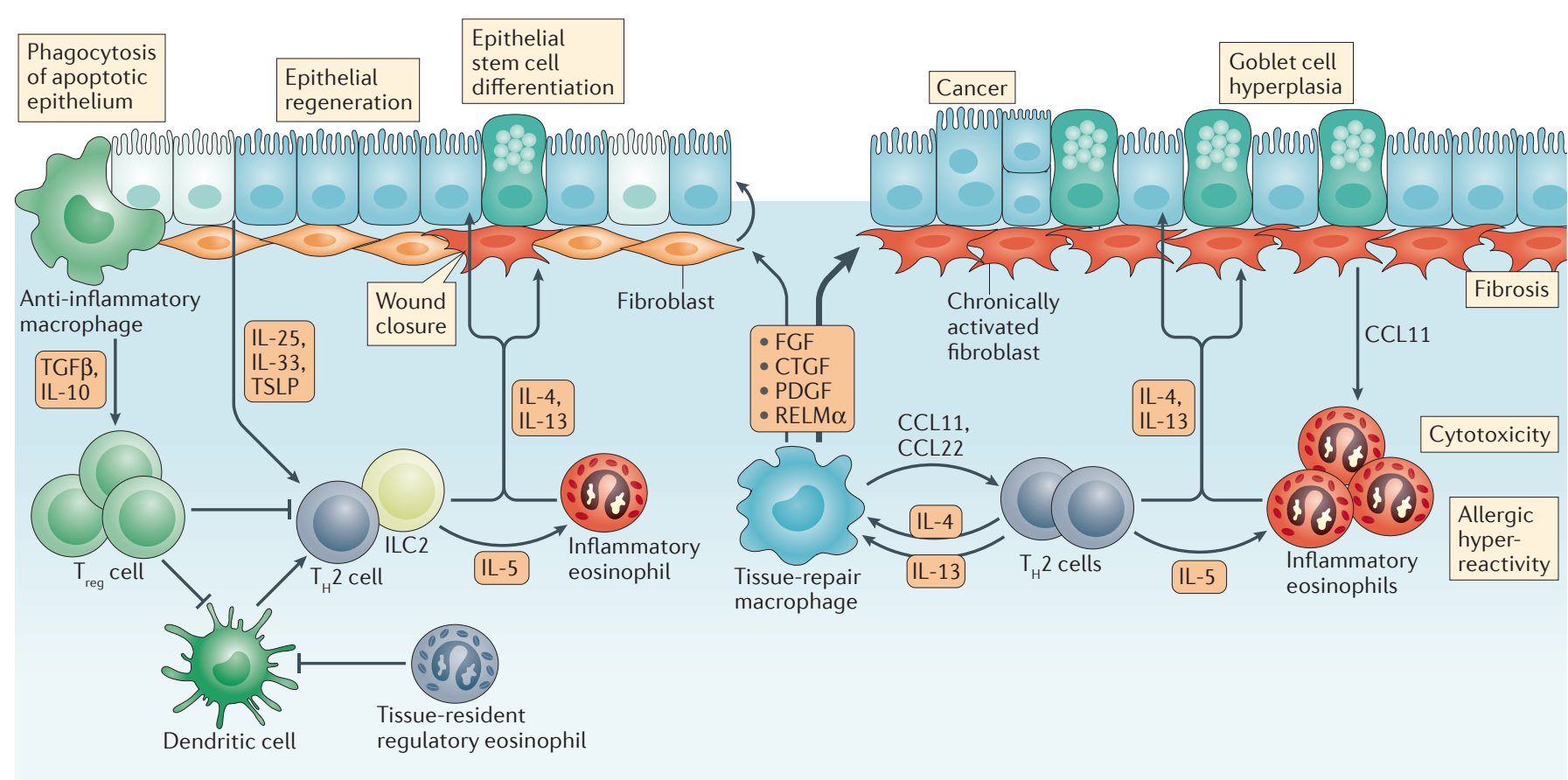

Type 2 polarization

Successful

wound healing
Type 2 damage and fibrosis

Figure 3 | Role of accessory cells in wound repair and type 2 fibrosis. Accessory cells, including macrophages, eosinophils, fibroblasts and epithelial cells, determine whether type 2 responses result in successful wound healing and tissue regeneration or progressive fibrosis and tissue pathology. In response to IL-4 and IL-13 signalling, epithelial cells proliferate and restore barrier function following injury. These cytokines also promote epithelial stem cell differentiation into various specialized cell types, including tuft cells and mucus-secreting goblet cells. In the case of highly polarized and chronic type 2 immune responses, biased differentiation and over-proliferation can result in goblet cell hyperplasia and excess mucus production in addition to predisposing individuals to cancers. Damaged epithelial cells also secrete alarmins, which can augment the type 2 response by increasing the expression of type 2 effector cytokines such as IL-4, IL-5 and IL-13. Eotaxins, such as CC-chemokine ligand 11 (CCL11), which are secreted from epithelial cells and fibroblasts in response to IL-13 signalling, recruit eosinophils. These eosinophils can augment epithelial regeneration by supplying a local source of IL-4 that triggers epithelial cells and other parenchymal cells to proliferate. Some populations of resident eosinophils can also dampen excessive type 2 responses by blocking antigen presentation and activation of Thelper 2 $\left(\mathrm{T}_{\mathrm{H}} 2\right)$ cells. Nevertheless, in highly polarized responses, excessive inflammatory eosinophilia can result in cytotoxicity, allergic hyperreactivity and fibrosis. In direct response to IL-4 and IL-13, fibroblasts secrete collagens and adopt contractile phenotypes, which can aid in wound closure. Furthermore, growth factors such as amphiregulin can aid in re-epithelialization of the wound surface. However, excessive and repetitive type 2 signalling in fibroblasts results in the excessive deposition of extracellular matrix, ultimately leading to scarring and fibrosis. Throughout this process, macrophages play a key regulatory role by either augmenting or suppressing the type 2 response at various stages. Tissue repair macrophages provide key growth factors, which both aid in wound repair and promote fibrogenesis. Furthermore, these cells can recruit $\mathrm{T}_{H} 2$ effector cells, which further polarize the response. Anti-inflammatory macrophages can dampen this response by providing IL-10 and transforming growth factor $\beta$ (TGF $\beta$ ), which promote regulatory T cell differentiation. Overall, the type 2 response is complex, targeting many cell types and having both beneficial and pathogenic features that are not mutually exclusive. During the development of therapeutics, how agonism or antagonism of various aspects of this response will affect the system as a whole will need to be carefully considered. CTGF, connective tissue growth factor; FGF, fibroblast growth factor; ILC2, group 2 innate lymphoid cell; PDGF, platelet-derived growth factor; RELM $\alpha$, resistin-like $\alpha$; $\mathrm{T}_{\text {reg }}$, regulatory $\mathrm{T}$; TSLP, thymic stromal lymphopoietin.

the pathogenesis of fibrosis. It was recently shown that hepatic stellate cells (resident liver fibroblasts) are able to recruit eosinophils through the upregulation of CCL11 (also known as eotaxin) in response to IL-13 signalling $^{188}$. Additionally, proliferating and migrating fibroblasts can activate pro-TGF $\beta$ through $\alpha_{v} \beta_{6}$ integrin and other integrin interactions that result in the generation of active TGF $\beta^{189}$. This pathway is critical in the pathogenesis of several models of hepatic, renal and pulmonary fibrosis ${ }^{190,191}$. However, it remains unclear whether integrin-mediated TGF $\beta$ activation plays a pathogenic pro-fibrotic role or a protective immunomodulatory role in highly polarized type 2 diseases; therefore, further studies are warranted. 
Surprisingly, recent work confirmed that IL-4 and/or IL-13 signalling directly through fibroblasts is necessary for the development of hepatic fibrosis during a highly polarized type 2 inflammatory response. $P d g f r b^{\text {cre }} I l 4 r a^{\text {Flox/Flox }}$ mice, which have fibroblasts deficient in IL-4Ra, failed to develop fibrosis in response to IL-13 (REF. 188). Because there was no decrease in the number of IL-4- and IL-13-activated macrophages in these studies, the authors also questioned the importance of pro-fibrotic IL-4- and IL-13-activated macrophages and intermediate factors such as TGF $\beta$ during highly polarized type 2 immune responses. Alternatively, the authors suggested that the pro-fibrotic functions of IL-4- and IL-13-activated macrophages are partially dependent on the prior activation of IL-4Ra-expressing fibroblasts by IL- 4 or IL-13. They also implied that strategies incorporating cell-specific blockade of IL- 4 or IL-13 signalling may prevent fibroblast-driven pathological fibrosis while maintaining the pro-regenerative and anti-inflammatory functions of type 2 immunity (FIG. 3).

Role of epithelial cells and other cell types. As mentioned above, the epithelia of the airways, gastrointestinal tract and liver are initiators of type 2 immunity via the secretion of alarmins. In contrast to alarmin expression, epithelial expression of chitin-degrading enzymes, including acidic mammalian chitinase (AMCase) and chitotriosidase 1 (CHIT1), has yielded mixed results. Early studies identified elevated levels of AMCase in the epithelial cells and macrophages of mice following allergen exposure and in humans with asthma ${ }^{192-194}$. Blockade of AMCase with anti-AMCase sera or allosamidin inhibited ovalbumin (OVA)-induced airway inflammation and IL-13 secretion in mice, suggesting that AMCase was required for allergen-induced airway inflammation ${ }^{193}$. Although administration of chitin can induce allergic responses that can be prevented by administration of AMCase ${ }^{195,196}$, Fitz and colleagues did not find any apparent role for AMCase in controlling the severity of allergic airway disease using HDM-, OVA- or cockroach allergen-induced airway inflammation in combination with either a small-molecule inhibitor targeting AMCase or AMCase deficiency in mice ${ }^{197}$. Nevertheless, AMCase-deficient mice exhibited impaired type 2 responses with a concomitant increase in neutrophilia and lymphocytic infiltration that compensated for the lack of type 2 effector function. In agreement with these studies, we observed an initial defect in type 2 effector functions during acute HDM challenge but found no significant impact on the progression of disease during chronic HDM exposure ${ }^{198}$. By contrast, we observed a profound impact on type 2 immune responses in the gastrointestinal tract in AMCase-deficient mice following intestinal helminth infection. Interestingly, these effects seemed to be most prominent in older mice ${ }^{199}$. A recent study also suggested that impaired clearance of chitin might contribute to the persistent activation of type 2-associated fibrogenic pathways in the lungs of aged mice and humans with interstitial lung diseases that exhibit epithelial cell dysfunction and reduced AMCase activity, but not CHIT1 activity ${ }^{199}$. It therefore appears that in response to chitin-rich microorganisms or allergens, potent type 2 immune responses can be invoked, in which chitinases may regulate the degree of inflammation. It remains undetermined to what degree AMCase and CHIT1 contribute to common allergen-driven inflammation.

In addition to AMCase and the alarmins, epithelial cell-derived TGF $\beta 1$ is a surprising but important type 2-promoting growth factor whose expression is elicited by IL-33 (REF. 200). Following exposure to allergens from Alternaria fungi, TGF $\beta$ R2-expressing ILC2s were recruited to TGF $\beta 1$-secreting epithelial cells, forming early innate inflammatory foci and propagating type 2-mediated airway inflammation and airway hyperreactivity. Local epithelial cell-derived TGF $\beta 1$ can also contribute to subepithelial fibroblast activation and airway remodelling ${ }^{201}$, placing epithelial cell-derived TGF $\beta 1$ and ILC2-derived IL-13 in an innate inflammatory and fibrotic loop. A recent study by Van Dyken and colleagues showed that tissue-derived signals, including the alarmins, provide a critical tissue-derived checkpoint, limiting the terminal differentiation and activation of both $\mathrm{T}_{\mathrm{H}} 2$ cells and ILC $2 \mathrm{~s}^{134}$. In line with previous studies ${ }^{115}$, inhibition of all three alarmins was required to dampen $\mathrm{T}_{\mathrm{H}} 2$ cell-mediated tissue inflammation ${ }^{134}$.

In addition to being a key source of important type 2-initiating cytokines, epithelial cells can respond to IL-4 and IL-13 directly, inducing important type 2 functions such as mucus secretion ${ }^{202}$. It was also recently shown that epithelial cells expressing IL-4Ra proliferate in response to IL- 4 and IL-13 and, furthermore, that IL-4 and IL-13 contribute to the proliferation and differentiation of adult tissue stem and progenitor cells.

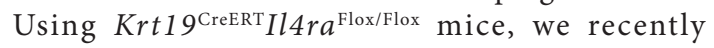
demonstrated that biliary proliferation, which accompanies fibrosis during schistosomiasis or during experimental fibrosis induced by IL-13 overexpression, is completely abolished when epithelial cells lack IL- $4 \mathrm{R}^{188}$. We also observed the biased differentiation of hepatic progenitor cells towards a cholangiocyte fate in response to IL-4 or IL-13 stimulation. Similarly, several groups have elucidated feedforward loops in the gut involving epithelial-derived IL-25 inducing IL-13 production by ILC2s, with IL-13 subsequently signalling through LGR $5^{+}$intestinal stem cells and/or their progeny. In response, these cells proliferate and exhibit differentiation biased towards tuft and goblet cell fates, enhancing parasite detection and expulsion ${ }^{83-85}$. Others have shown that fibroblast-derived IL-33 can directly target these stem cells and enhance barrier immunity against Salmonella infection ${ }^{203}$. Whether this ILC2-tuft cell feedforward loop also operates in the lung or any other organ is unclear. Nevertheless, these examples illustrate that type 2 signalling through the epithelium and epithelial stem cells plays important roles in epithelial regeneration and the maintenance of barrier function during the course of injury. When designing therapies that aim to block the fibroproliferative effects of type 2 signalling through systemic cytokine or receptor blockade, the potential adverse effects that could result due to impaired epithelial regeneration and function should be considered (FIG. 3). 


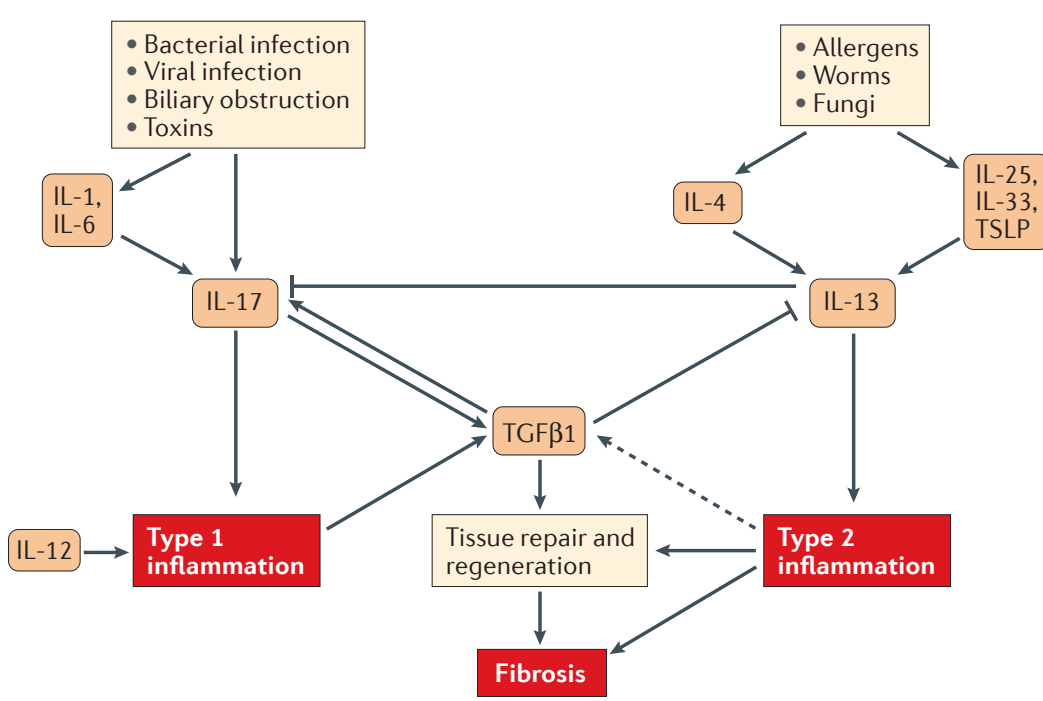

Figure 4 | Crosstalk between core immunological pathways of fibrosis. Two key immunological mechanisms driving tissue repair and fibrosis have been described, and both pathways exhibit cross-regulatory activity. Bacterial and viral infections as well as toxic substances like bleomycin and carbon tetrachloride have been shown to activate the IL-1-IL-17-transforming growth factor $\beta$ (TGF $\beta$ ) axis, which is believed to be a major driver of tissue repair and fibrosis during sustained type 1- and Thelper 17 cell-driven inflammatory responses. TGF $\beta$ has feedback anti-inflammatory activity and simultaneously activates extracellular matrix production by myofibroblasts. IL-13, by contrast, has been identified as the major driver of tissue repair and fibrosis during sustained type 2 responses induced by allergens, worms and some fungi. The two pathways also exhibit substantial negative regulatory activity, as depicted in the figure. TSLP, thymic stromal lymphopoietin.

\section{Cross-regulation of fibrotic pathways}

Type 2 immunity is tightly regulated by several distinct mechanisms (as recently reviewed ${ }^{4}$ ), which help to prevent complications resulting from sustained type 2 signalling. A number of type 1 -associated cytokines are potent inhibitors of type 2 immunity through their effects on naive $\mathrm{CD} 4^{+} \mathrm{T}$ cell differentiation. In particular, IL-12 signalling prohibits the differentiation of the $\mathrm{T}_{\mathrm{H}} 2$ cell lineage by promoting the differentiation of naive $\mathrm{T}$ cells towards a $\mathrm{T}_{\mathrm{H}} 1$ cell phenotype. IFN $\gamma$ activated macrophages upregulate IL-12 and suppress IL-10 expression, further shifting naive helper T cells towards a $\mathrm{T}_{\mathrm{H}} 1$ or $\mathrm{T}_{\mathrm{H}} 17$ cell fate ${ }^{204-207}$. IL-10 and TGF $\beta$ produced by $\mathrm{DCs}$ and regulatory $\mathrm{T}$ cells can also actively suppress both type 1 and type 2 responses, thereby inhibiting excessive cytokine production ${ }^{208,209}$.

Additionally, the $\mathrm{T}_{\mathrm{H}} 2$ effector cytokine IL-13 engages an inducible, high-affinity decoy receptor, IL-13Ra2, which suppresses IL-13 effector function by sequestering the protein away from the IL-4R $\alpha-\mathrm{IL}-13 \mathrm{R} \alpha 1$ signalling receptor complex ${ }^{210,211}$. Interestingly, these suppressive mechanisms have functional redundancy and work in collaboration to control excessive type 2 immunity ${ }^{212}$. Recent studies demonstrated that several type 1- and $\mathrm{T}_{\mathrm{H}} 17$-derived cytokines can drive the production of IL-13R $\alpha 2$, suggesting potent cross-regulation of IL-13 effector function by type 1 - and $\mathrm{T}_{\mathrm{H}} 17$-driven immunity. Indeed, it has been shown that several cytokine combinations, including TNF and IL-4 or IL-17, can synergize to drive high levels of IL-13Ra2 expression, blocking the ability of IL-13 to upregulate downstream targets such as CCL26 in both human and mouse fibroblasts ${ }^{213}$.

In addition to the primary role of IL-13Ra2 as a decoy receptor for IL-13, Elias and colleagues have reported that chitinase-3-like protein 1 (CHI3L1) may signal through IL-13Ra2 (REF. 214). Recently, a binding partner of the CHI3L1-IL-13Ra2 complex, called insulin-like growth factor-binding protein 3 receptor (IGFBP3R, also known as TMEM219), was identified. It has been suggested that IGFBP3R regulates the signalling properties of the CHI3L1-IL-13Ra2 complex and augments the decoy functions of IL-13R $\alpha 2$ in addition to controlling diverse pathways regulating TGF $\beta 1$ production, antibacterial responses, inflammasome activation, oxidant injury and apoptosis ${ }^{215}$. Nevertheless, independent validation and replication of these studies are necessary.

Regulation of programmed cell death, whether apoptosis, necroptosis or pyroptosis, may be an additional core pathway modulating remodelling and fibrotic responses. Whether too much or too little, the rate and relevance of cell death with respect to disease initiation and progression continue to be debated. Damage to or induced senescence of alveolar type 2 cells is considered to be one of the earliest pathological events in IPF ${ }^{216,217}$, and it has been suggested that senescence may be linked to low-level ongoing inflammation and thus may be a key initiator of fibrosis (as recently reviewed ${ }^{218}$ ). Indeed, experimental administration of an anti-Fas antibody ${ }^{219}$ or bleomycin ${ }^{220}$, which cause epithelial cell damage, apoptosis and cell death, is frequently used as a model of pulmonary fibrosis; this finding implicates epithelial cell impairment, including both senescence and cell death, in the pathogenesis of pulmonary fibrosis. In addition, fibroblasts isolated from patients with IPF appear to have a slower growth rate along with increased activation and release of pro-fibrotic mediators yet show increased rates of apoptosis ${ }^{221}$. In regions of epithelial cell death, increases in myofibroblast activation have been observed, presumably for repair of the damaged epithelium ${ }^{222}$. Similarly, apoptosis of endothelial cells with the release of CTGF can also contribute to fibrogenesis ${ }^{223}$, suggesting that local tissue damage and pervasive cell death may be important drivers of fibrosis. In response to such tissue damage and apoptosis, both inflammatory ${ }^{224}$ and anti-inflammatory pathways have been identified, with increased secondary apoptosis ${ }^{224}$ reducing tissue build-up ${ }^{225,226}$. In particular, the phagocytosis of apoptotic epithelial cells, either by neighbouring epithelial cells ${ }^{87,227}$ or by professional phagocytes ${ }^{28,229}$, activates distinct anti-inflammatory responses that limit intestinal and airway inflammation. Additionally, engagement of apoptotic cell sensors may be a co-requisite, along with IL-4 and IL-13 signalling, to fully potentiating the activation of tissue repair macrophages ${ }^{168}$. Together, these data suggest that cell death may be a core fibrotic pathway, both propagating disease through mediator release and resolving local tissue damage by regulating inflammation and thus reflecting the cyclic paradigm of wound healing that may lead to fibrosis when chronically activated or dysregulated (FIG. 4). 

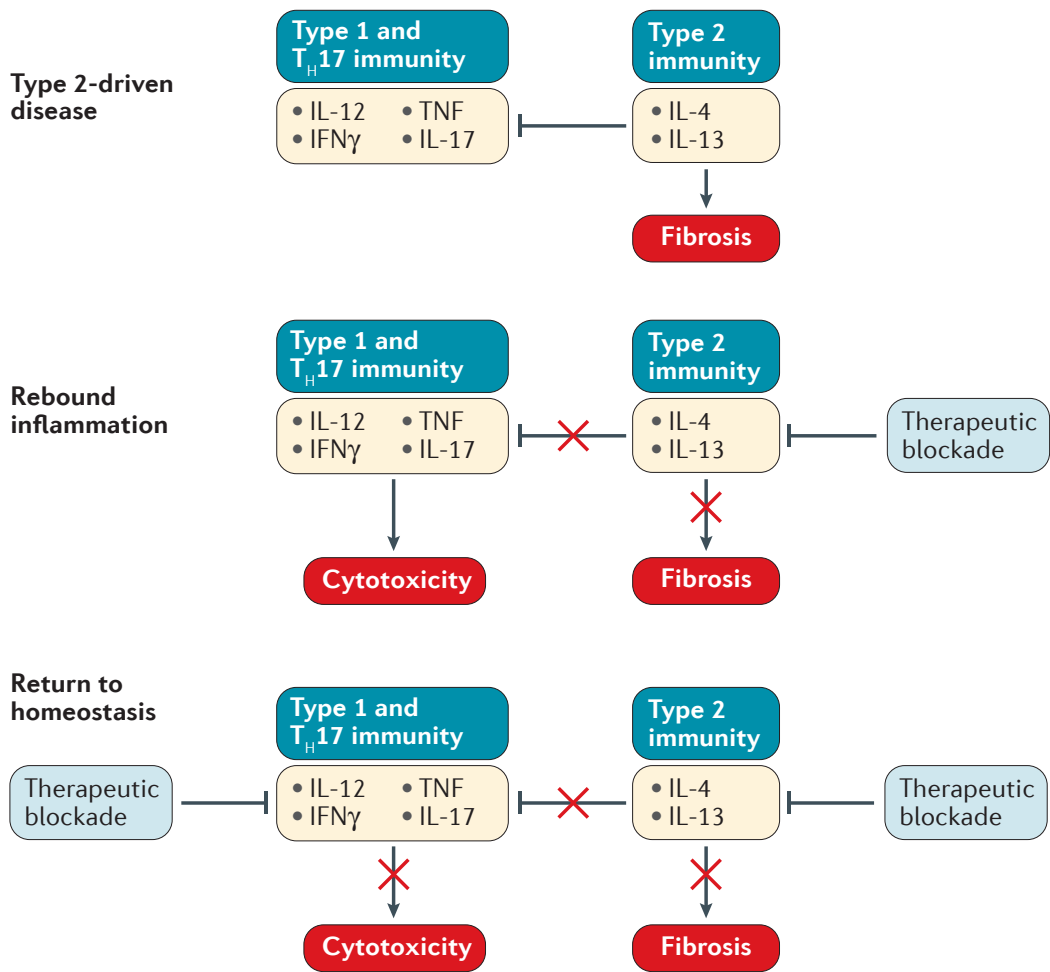

Figure 5 | Rebound inflammation during therapeutic intervention. Following therapeutic blockade of type 2 immunity, important cross-regulatory networks are dysregulated, allowing type 1- and/or T helper $17\left(T_{H} 17\right)$ cell-driven inflammation to rebound and exposing subjects to potentially harmful cytotoxic side effects. Successful therapeutics should return patients to a healthy homeostatic set point, perhaps by simultaneously targeting the pathogenic actions of sustained type 1 - and/or $T_{H} 17$-driven and type 2 inflammation. IFN $\gamma$, interferon $\gamma$; TNF, tumour necrosis factor.

\section{Future directions and opportunities}

Given the extensive evidence demonstrating the profibrotic nature of type 2 immunity and, in particular, IL-4 and IL-13 signalling when continuously activated, this type of immune response has proven attractive for therapeutic modulation during the course of various allergic and fibrotic diseases. Several groups have investigated or are actively investigating blockade using antibodies and/or antagonists targeting various aspects of the IL-4 and IL-13 signalling pathways alone or in combination using bifunctional or bi-specific strategies. The results of such trials in various diseases have been mixed, and in some cases, the trials have demonstrated worsened outcomes in patients receiving treatment compared with control arms ${ }^{74,79,80}$.

As stated earlier, several type 1 cytokines have been shown to counter-regulate type 2 immunity. However, type 2 cytokines are also important regulators of type 1and $\mathrm{T}_{\mathrm{H}} 17$-driven inflammatory responses. Recently, several studies have revealed that blockade of type 2 cytokines can dysregulate this cross-regulatory mechanism and promote type 1- and $\mathrm{T}_{\mathrm{H}} 17$-driven inflammation, exacerbating IFN $\gamma$ - and IL-17A-driven cytotoxic injury. During the course of experimental murine schistosomiasis and in pulmonary granuloma models, blocking IL-13 alone significantly reduces fibrosis but concurrently leads to increases in IFN $\gamma$ production and subsequent increases in TNF production and inflammatory tissue necrosis, exacerbating liver and lung damage. However, dual blockade of IL-13 and IFN $\gamma$ led to a marked reduction in fibrosis and eliminated the rebound type 1 inflammation and associated damage observed in mice treated with anti-IL-13 alone ${ }^{230}$. Similarly, it has been shown that blockade of IL-4 and/or IL-13 in HDM-induced allergy models induces substantial $\mathrm{T}_{\mathrm{H}} 17$ cell-driven neutrophilic inflammation; however, dual blockade of IL-13 and IL-17A protected mice from both eosinophilic and neutrophilic inflammation and eliminated associated mucus production and airway hyperreactivity, illustrating the benefits of dual- or multi-blockade strategies to combat rebound inflammation ${ }^{230,231}$ (FIG. 5). It is interesting to speculate that some of the disappointing outcomes observed in clinical trials blocking aspects of the IL-4 and/or IL-13 signalling pathways may be the result of unintended dysregulation of the type 1- and $\mathrm{T}_{\mathrm{H}} 17$-driven inflammatory responses and/or disruption of important beneficial aspects of IL-4 and IL-13 signalling, such as epithelial regeneration. Together, these studies suggest that careful targeting and dosing of therapeutics will be necessary to successfully block the pathogenic features of sustained type 2-driven inflammation without sacrificing the beneficial features of wound repair and epithelial regeneration or subjecting patients to potentially harmful rebound inflammatory responses. Uncovering the various cellular, molecular and genetic mechanisms that sustain pro-fibrotic signalling and inhibit endogenous mechanisms to end wound healing responses will be an important research goal in the coming years.
1. Nakayama, T. et al. Th2 cells in health and disease. Annu. Rev. Immunol. 35, 53-84 (2017).

2. Mosmann, T. R., Cherwinski, H., Bond, M. W., Giedlin, M. A. \& Coffman, R. L. Two types of murine helper T cell clone. I. Definition according to profiles of lymphokine activities and secreted proteins. J. Immunol. 136, 2348-2357 (1986).

3. Abbas, A. K., Murphy, K. M. \& Sher, A. Functional diversity of helper T lymphocytes. Nature 383 787-793 (1996).

4. Wynn, T. A. Type 2 cytokines: mechanisms and therapeutic strategies. Nat. Rev. Immunol. 15, 271-282 (2015)

5. Minutti, C. M., Knipper, J. A., Allen, J. E. \& Zaiss, D. M. Tissue-specific contribution of macrophages to wound healing. Semin. Cell Dev. Biol. 61, 3-11 (2017).

6. Van Dyken, S. J. \& Locksley, R. M. Interleukin-4- and interleukin-13-mediated alternatively activated macrophages: roles in homeostasis and disease. Annu. Rev. Immunol. 31, 317-343 (2013).
7. Wynn, T. A. $\&$ Vannella, K. M. Macrophages in tissue repair, regeneration, and fibrosis. Immunity $\mathbf{4 4 ,}$ 450-462 (2016).

8. McSorley, H. J. \& Maizels, R. M. Helminth infections and host immune regulation. Clin. Microbiol. Rev. 25 585-608 (2012).

9. Colley, D. G., Bustinduy, A. L., Secor, W. E. \& King, C. H. Human schistosomiasis. Lancet 383 2253-2264 (2014)

10. Pearce, E. J. \& MacDonald, A. S. The immunobiology of schistosomiasis. Nat. Rev. Immunol. 2, 499-511 (2002).

11. Fairfax, K., Nascimento, M., Huang, S. C., Everts, B. \& Pearce, E. J. Th2 responses in schistosomiasis. Seminars Immunopathol. 34, 863-871 (2012).

12. Sripa, B., Kaewkes, S., Intapan, P. M., Maleewong, W. $\&$ Brindley, P. J. Food-borne trematodiases in Southeast Asia epidemiology, pathology, clinical manifestation and control. Adv. Parasitol. 72 , 305-350 (2010).
13. Sripa, B. et al. Liver fluke induces cholangiocarcinoma PLoS Med. 4, e201 (2007).

14. Sripa, B. et al. The tumorigenic liver fluke Opisthorchis viverrini-multiple pathways to cancer. Trends Parasitol. 28, 395-407 (2012).

15. Chen, Y. et al. Coinfection with Clonorchis sinensis modulates murine host response against Trichinella spiralis infection. Parasitol. Res. 112, 3167-3179 (2013).

16. Xu, Y. Q. et al. Interleukin-13 is involved in the formation of liver fibrosis in Clonorchis sinensis-infected mice. Parasitol. Res. 115, 2653-2660 (2016).

17. Zhang, B. B. et al. Increased hepatic Th2 and Treg subsets are associated with biliary fibrosis in different strains of mice caused by Clonorchis sinensis. PLOS ONE 12, e0171005 (2017).

18. Landi, A. et al. Differential serum levels of eosinophilic eotaxins in primary sclerosing cholangitis, primary biliary cirrhosis, and autoimmune hepatitis. J. Interferon Cytokine Res. 34, 204-214 (2014). 
19. Li, J. et al. Th2 signals induce epithelial injury in mice and are compatible with the biliary atresia phenotype. J. Clin. Invest. 121, 4244-4256 (2011)

20. Michalopoulos, G. K. \& DeFrances, M. C. Liver regeneration. Science 276, 60-66 (1997).

21. Kotton, D. N. \& Morrisey, E. E. Lung regeneration: mechanisms, applications and emerging stem cell populations. Nat. Med. 20, 822-832 (2014).

22. Boulet, L. P. \& Boulay, M. E. Asthma-related comorbidities. Expert Rev. Respir. Med. 5, 377-393 (2011).

23. Chatila, W. M., Thomashow, B. M., Minai, O. A., Criner, G. J. \& Make, B. J. Comorbidities in chronic obstructive pulmonary disease. Proc. Am. Thorac. Soc 5, 549-555 (2008).

24. Yanagi, S., Tsubouchi, H., Miura, A., Matsumoto, N. \& Nakazato, M. Breakdown of epithelial barrier integrity and overdrive activation of alveolar epithelial cells in the pathogenesis of acute respiratory distress syndrome and lung fibrosis. Biomed. Res. Int. 2015, 573210 (2015).

25. Payne, D. N. et al. Early thickening of the reticular basement membrane in children with difficult asthma. Am. J. Respir. Crit. Care Med. 167, 78-82 (2003)

26. Mineo, G. et al. Post-ARDS pulmonary fibrosis in patients with H1N1 pneumonia: role of follow-up CT. Radiol Med. 117, 185-200 (2012).

27. Arakawa, H. et al. Asbestosis and other pulmonary fibrosis in asbestos-exposed workers: high-resolution CT features with pathological correlations. Eur. Radio 26, 1485-1492 (2016).

28. Aceves, S. S. \& Broide, D. H. Airway fibrosis and angiogenesis due to eosinophil trafficking in chronic asthma. Curr. Mol. Med. 8, 350-358 (2008).

29. Williams, E. C. Case of pulmonary fibrosis following a tuberculous infection. Proc. R. Soc. Med. 16, 79 (1923).

30. Churg, A., Muller, N. L. \& Wright, J. L. Respiratory bronchiolitis/interstitial lung disease: fibrosis, pulmonary function, and evolving concepts. Arch. Pathol. Lab Med. 134, 27-32 (2010).

31. Snider, G. L. Perivascular fibrosis of muscular pulmonary arteries in COPD. Chest 105, 973-974 (1994).

32. Harris, W. T. et al. Myofibroblast differentiation and enhanced TGF-B signaling in cystic fibrosis lung disease. PLOS ONE 8, e70196 (2013).

33. Sprague, H. B. et al. Pulmonary fibrosis and emphysema, with slight bronchiectasis. N. Engl. J. Med. 238, 667-671 (1948).

34. Griffin, J. P. Interstitial pulmonary fibrosis and lung cancer. Chest 108, 1193-1194 (1995).

35. Auerbach, S. H., Mims, O. M. \& Goodpasture, E. W. Pulmonary fibrosis secondary to pneumonia. Am. J. Pathol. 28, 69-87 (1952).

36. Emirgil, C., Sobol, B. J., Herbert, W. H. \& Trout, K. The lesser circulation in pulmonary fibrosis secondary to sarcoidosis and its relationship to respiratory function. Chest 60, 371-378 (1971)

37. Kaviratne, M. et al. IL-13 activates a mechanism of tissue fibrosis that is completely TGF- $\beta$ independent. J. Immunol. 173, 4020-4029 (2004).

38. Wilson, M. S. et al. Bleomycin and IL-1 $\beta$-mediated pulmonary fibrosis is IL-17A dependent. J. Exp. Med. 207, 535-552 (2010).

39. Simonian, P. L. et al. Th17-polarized immune response in a murine model of hypersensitivity pneumonitis and lung fibrosis. J. Immunol. 182, 657-665 (2009)

40. Braun, R. K. et al. IL-17 producing $\gamma \delta$ T cells are required for a controlled inflammatory response after bleomycin-induced lung injury. Inflammation $\mathbf{3 1}$, 167-179 (2008)

41. Li, J. T. et al. Unexpected role for adaptive $\alpha \beta$ Th 17 cells in acute respiratory distress syndrome. J. Immunol. 195, 87-95 (2015)

42. Ferro, A., Zebedeo, C. N., Davis, C., Ng, K. W. \& Pfau, J. C. Amphibole, but not chrysotile, asbestos induces anti-nuclear autoantibodies and IL-17 in C57BL/6 mice. J. Immunotoxicol 11, 283-290 (2014).

43. Zhang, J. et al. Increased expression of CD4+ IL-17 cells in the lung tissue of patients with stable chronic obstructive pulmonary disease (COPD) and smokers. Int. Immunopharmacol. 15, 58-66 (2013).

44. Chan, Y. R. et al. Patients with cystic fibrosis have inducible IL- $17^{+} \mathrm{IL}-22^{+}$memory cells in lung draining lymph nodes. J. Allergy Clin. Immunol. 131 1117-1129 (2013)

45. Khader, S. A. et al. IL-23 compensates for the absence of IL-12p70 and is essential for the IL-17 response during tuberculosis but is dispensable for protection and antigen-specific IFN- $\gamma$ responses if IL- $12 \mathrm{p} 70$ is available. J. Immunol. 175, 788-795 (2005).
46. McAllister, F. et al. Role of IL-17A, IL-17F, and the $\mathrm{IL}-17$ receptor in regulating growth-related oncogenealpha and granulocyte colony-stimulating factor in bronchial epithelium: implications for airway inflammation in cystic fibrosis. J. Immunol. 175 , 404-412 (2005).

47. Wong, C. K. et al. Proinflammatory cytokines (IL-17, IL-6, IL-18 and IL-12) and Th cytokines (IFN- $\gamma$, IL-4 IL-10 and IL-13) in patients with allergic asthma. Clin. Exp. Immunol. 125, 177-183 (2001).

48. Baurakiades, E. et al The roles of ADAM33, ADAM28, IL-13 and IL-4 in the development of lung injuries in children with lethal non-pandemic acute infectious pneumonia. J. Clin. Virol. 61, 585-589 (2014).

49. Heitmann, L. et al. The IL-13/IL-4R $\alpha$ axis is involved in tuberculosis-associated pathology. J. Pathol. 234 338-350 (2014)

50. Keane, M. P. et al. IL-13 is pivotal in the fibroobliterative process of bronchiolitis obliterans syndrome. J. Immunol. 178, 511-519 (2007).

51. van der Pouw Kraan, T. C. et al. Chronic obstructive pulmonary disease is associated with the - 1055 IL-13 promoter polymorphism. Genes Immun. 3, 436-439 (2002)

52. Wills-Karp, M. et al. Interleukin-13: central mediator of allergic asthma. Science 282, 2258-2261 (1998)

53. Kolodsick, J. E. et al. Protection from fluorescein isothiocyanate-induced fibrosis in IL-13-deficient, but not IL-4-deficient, mice results from impaired collagen synthesis by fibroblasts. J. Immunol. 172 4068-4076 (2004)

54. Jakubzick, C. et al. Therapeutic attenuation of pulmonary fibrosis via targeting of IL-4- and LL-13-responsive cells. J. Immunol. 171, 2684-2693 (2003).

55. Belperio, J. A. et al. Interaction of IL-13 and C10 in the pathogenesis of bleomycin-induced pulmonary fibrosis. Am. J. Respiratory Cell. Mol. Biol. 27, 419-427 (2002).

56. Zhu, Z. et al. Pulmonary expression of interleukin-13 causes inflammation, mucus hypersecretion subepithelial fibrosis, physiologic abnormalities, and eotaxin production. J. Clin. Invest. 103, 779-788 (1999).

57. Grunig, G. et al. Requirement for IL-13 independently of IL-4 in experimental asthma. Science $\mathbf{2 8 2}$, 2261-2263 (1998)

58. Kasaian, M. T. \& Miller, D. K. IL-13 as a therapeutic target for respiratory disease. Biochem. Pharmacol. 76, 147-155 (2008).

59. Piper, E. et al. A phase II placebo-controlled study of tralokinumab in moderate-to-severe asthma. Eur. Respir. J. 41, 330-338 (2013).

60. Corren, J et al. Lebrikizumab treatment in adults with asthma. N. Engl. J. Med. 365, 1088-1098 (2011).

61. Wenzel, S. et al. Dupilumab in persistent asthma with elevated eosinophil levels. N. Engl. J. Med. 368 2455-2466 (2013)

62. Park, S. W. et al. Interleukin-13 and its receptors in idiopathic interstitial pneumonia: clinical implications for lung function. J. Kor. Med. Sci. 24, 614-620 (2009)

63. Murray, L. A. et al. Hyper-responsiveness of IPF/UIP fibroblasts: interplay between TGF $\beta 1, \mathrm{IL}-13$ and CCL2. Int. J. Biochem. Cell Biol. 40, 2174-2182 (2008)

64. Jakubzick, C. et al. Human pulmonary fibroblasts exhibit altered interleukin-4 and interleukin-13 receptor subunit expression in idiopathic interstitial pneumonia. Am. J. Pathol. 164, 1989-2001 (2004).

65. Hancock, A., Armstrong, L., Gama, R. \& Millar, A. Production of interleukin 13 by alveolar macrophages from normal and fibrotic lung. Am. J. Respiratory Cell. Mol. Biol. 18, 60-65 (1998).

66. Murray, L. A. et al. Targeting interleukin-13 with tralokinumab attenuates lung fibrosis and epithelial damage in a humanized SCID idiopathic pulmonary fibrosis model. Am. J. Respiratory Cell. Mol. Biol. 50 985-994 (2014)

67. Fichtner-Feigl, S., Strober, W., Kawakami, K., Puri, R. K. \& Kitani, A. IL-13 signaling through the IL-13 $\alpha 2$ receptor is involved in induction of TGF- $\beta$ production and fibrosis. Nat. Med. 12, 99-106 (2006).

68. Lee, C. G. et al. Interleukin-13 induces tissue fibrosis by selectively stimulating and activating transforming growth factor $\beta_{1}$. J. Exp. Med. 194, 809-821 (2001).

69. Deshpande, V. et al. Consensus statement on the pathology of IgG4-related disease. Mod. Pathol. 25 1181-1192 (2012).
70. Khosroshahi, A. et al. Rituximab for the treatment of IgG4-related disease: lessons from 10 consecutive patients. Med. (Baltimore) 91, 57-66 (2012).

71. Bieber, T. Atopic dermatitis. N. Engl. J. Med. 358 1483-1494 (2008).

72. Salimi, M. et al. A role for IL-25 and IL-33-driven type-2 innate lymphoid cells in atopic dermatitis. J. Exp. Med. 210, 2939-2950 (2013).

73. Imai, Y. et al. Skin-specific expression of IL-33 activates group 2 innate lymphoid cells and elicits atopic dermatitis-like inflammation in mice. Proc. Nat Acad. Sci. USA 110, 13921-13926 (2013).

74. Beck, L. A. et al. Dupilumab treatment in adults with moderate-to-severe atopic dermatitis. N. Engl. J. Med. 371, 130-139 (2014).

75. Danese, S. \& Fiocchi, C. Ulcerative colitis. N. Engl. J. Med. 365, 1713-1725 (2011).

76. Neurath, M. F. Cytokines in inflammatory bowe disease. Nat. Rev. Immunol. 14, 329-342 (2014).

77. Fuss, I. J. \& Strober, W. The role of IL-13 and NK T cells in experimental and human ulcerative colitis. Mucosal Immunol. 1 (Suppl. 1), S31-S33 (2008).

78. Heller, F. et al. Interleukin-13 is the key effector Th2 cytokine in ulcerative colitis that affects epithelial tight junctions, apoptosis, and cell restitution. Gastroenterology 129, 550-564 (2005).

79. Danese, S. et al. Tralokinumab for moderate-to-severe UC: a randomised, double-blind, placebo-controlled, phase lla study. Gut 64, 243-249 (2015)

80. Reinisch, W. et al. Anrukinzumab, an anti-interleukin 13 monoclonal antibody, in active UC: efficacy and safety from a phase lla randomised multicentre study. Gut 64, 894-900 (2015)

81. Giacomin, P. R. et al. Epithelial-intrinsic IKK $\alpha$ expression regulates group 3 innate lymphoid cell responses and antibacterial immunity. J. Exp. Med. 212, 1513-1528 (2015)

82. Zaph, C. et al. Epithelial-cell-intrinsic IKK- $\beta$ expression regulates intestinal immune homeostasis. Nature 446, 552-556 (2007).

83. Gerbe, F. et al. Intestinal epithelial tuft cells initiate type 2 mucosal immunity to helminth parasites. Nature 529, 226-230 (2016)

This study identified tuft cells as a critical source of the IL-25 that drives IL-4 and IL-13 production during intestinal helminth infection.

84. Howitt, M. R. et al. Tuft cells, taste-chemosensory cells, orchestrate parasite type 2 immunity in the gut Science 351, 1329-1333 (2016).

This study identified the chemo-sensing proteins gustducin and TRPM5 as components crucial for DCLK1 + tuft cells to sense parasites and secrete IL-25.

85. von Moltke, J., Ji, M., Liang, H. E. \& Locksley, R. M. Tuft-cell-derived IL-25 regulates an intestinal ILC2-epithelial response circuit. Nature 529 221-225 (2016)

This study identified tuft cell-derived IL-25 as crucial to intestinal ILC2 homeostasis and to biasing of stem cell differentiation towards a tuft cell fate.

86. Knoop, K. A., McDonald, K. G., McCrate, S., McDole, J. R. \& Newberry, R. D. Microbial sensing by goblet cells controls immune surveillance of luminal antigens in the colon. Mucosal Immunol. 8, 198-210 (2015).

87. Juncadella, I. J. et al. Apoptotic cell clearance by bronchial epithelial cells critically influences airway inflammation. Nature 493, 547-551 (2013).

88. Fort, M. M. et al. IL-25 induces IL-4, IL-5, and IL-13 and Th2-associated pathologies in vivo. Immunity 15 985-995 (2001).

89. Hurst, S. D. et al. New IL-17 family members promote Th1 or Th2 responses in the lung: in vivo function of the novel cytokine IL-25. J. Immunol. 169, 443-453 (2002).

90. Swaidani, S. et al. The critical role of epithelial-derived Act 1 in IL-17- and IL-25-mediated pulmonary inflammation. J. Immunol. 182, 1631-1640 (2009).

91. Schmitz, J. et al. IL-33, an interleukin-1-like cytokine that signals via the IL-1 receptor-related protein ST2 and induces $T$ helper type 2-associated cytokines. Immunity 23, 479-490 (2005).

92. Humphreys, N. E., Xu, D., Hepworth, M. R., Liew, F. Y. $\&$ Grencis, R. K. IL-33, a potent inducer of adaptive immunity to intestinal nematodes. J. Immunol. 180 2443-2449 (2008).

93. Komai-Koma, M. et al. IL-33 is a chemoattractant for human Th2 cells. Eur. J. Immunol. 37, 2779-2786 (2007). 
94. Allakhverdi, Z., Smith, D. E., Comeau, M. R. \& Delespesse, G. Cutting edge: the ST2 ligand IL-33 potently activates and drives maturation of human mast cells. J. Immunol. 179, 2051-2054 (2007)

95. Hams, E. et al. IL-25 and type 2 innate lymphoid cells induce pulmonary fibrosis. Proc. Natl Acad. Sci. USA 111, 367-372 (2014).

96. Yao, X. et al. Characteristics of IL-25 and allergeninduced airway fibrosis in a murine model of asthma. Respirology 20, 730-738 (2015).

97. Yanaba, K., Yoshizaki, A., Asano, Y., Kadono, T \& Sato, S. Serum IL-33 levels are raised in patients with systemic sclerosis: association with extent of skin sclerosis and severity of pulmonary fibrosis. Clin. Rheumatol 30, 825-830 (2011).

98. Rankin, A. L. et al. IL-33 induces IL-13-dependent cutaneous fibrosis. J. Immunol. 184, 1526-1535 (2010).

99. Gao, Y. et al. IL-33 treatment attenuated diet-induced hepatic steatosis but aggravated hepatic fibrosis. Oncotarget 7, 33649-33661 (2016).

100. Masterson, J. C. et al. Eosinophils and IL-33 perpetuate chronic inflammation and fibrosis in a pediatric population with stricturing crohn's ileitis Inflamm. Bowel Dis. 21, 2429-2440 (2015).

101. Roussel, L. Farias, R \& Rousseau, S. IL-33 is expressed in epithelia from patients with cystic fibrosis and potentiates neutrophil recruitment. J. Allergy Clin. Immunol. 131, 913-916 (2013).

102. Lopetuso, L. R., Scaldaferri, F. \& Pizarro, T T. Emerging role of the interleukin (IL)-33/ST2 axis in gut mucosal wound healing and fibrosis. Fibrogen. Tissue Repair 5, 18 (2012).

103. Li, D. et al. IL-33 promotes ST2-dependent lung fibrosis by the induction of alternatively activated macrophages and innate lymphoid cells in mice. J. Allergy Clin. Immunol. 134, 1422-1432 (2014).

104. Lee, J. U. et al. Upregulation of interleukin-33 and thymic stromal lymphopoietin levels in the lungs of idiopathic pulmonary fibrosis. BMC Pulm Med. 17, 39 (2017).

105. Herro, R., Da Silva Antunes, R., Aguilera, A. R. Tamada, K. \& Croft, M. Tumor necrosis factor superfamily 14 (LIGHT) controls thymic stromal lymphopoietin to drive pulmonary fibrosis. J. Allergy Clin. Immunol. 136, 757-768 (2015)

106. Truchetet, M. E. et al. Platelets induce thymic stromal lymphopoietin production by endothelial cells: contribution to fibrosis in human systemic sclerosis. Arthritis Rheumatol 68, 2784-2794 (2016).

107. Usategui, A. et al. A profibrotic role for thymic stroma lymphopoietin in systemic sclerosis. Ann. Rheum. Dis. 72, 2018-2023 (2013)

108. Christmann, R. B. et al. Thymic stromal lymphopoietin is up-regulated in the skin of patients with systemic sclerosis and induces profibrotic genes and intracellular signaling that overlap with those induced by interleukin-13 and transforming growth factor $\beta$. Arthritis Rheum. 65, 1335-1346 (2013).

109. Son A et al. TWEAK/Fn 14 pathway promotes a T helper 2-type chronic colitis with fibrosis in mice. Mucosal Immunol. 6, 1131-1142 (2013)

110. Oh, M. H. et al. IL-13 induces skin fibrosis in atopic dermatitis by thymic stromal lymphopoietin. J. Immunol. 186, 7232-7242 (2011).

111. Hsieh, P. F. et al. Treatment with cytokine thymic stromal lymphopoietin short hairpin RNA substantially reduces TGF- $\beta 1$-induced interstitial cellular fibrosis. Exp. Cell Res. 347, 153-160 (2016)

112. Li, L., Tang, S. \& Tang, X. Thymic stromal lymphopoietin promotes fibrosis and activates mitogen-activated protein kinases in MRC- 5 cells Med. Sci. Monit. 22, 2357-2362 (2016)

113. Shin, J. U. et al. TSLP is a potential initiator of collagen synthesis and an activator of CXCR4/SDF-1 axis in keloid pathogenesis. J. Invest. Dermatol. 136, 507-515 (2016)

114. Willart, M. A. et al. Interleukin- $1 \alpha$ controls allergic sensitization to inhaled house dust mite via the epithelial release of GM-CSF and IL-33. J. Exp. Med. 209, 1505-1517 (2012)

115. Vannella, K. M. et al. Combinatorial targeting of TSLP, IL-25, and IL-33 in type 2 cytokine-driven inflammation and fibrosis. Sci. Transl. Med. 8 , 337 ra65 (2016).

This study showed partial redundancy of the alarmin proteins in maintaining type 2 responses and that early combinatorial blockade may be efficacious in reducing disease severity.

116. Gibbs, B. F. et al. Purified human peripheral blood basophils release interleukin-13 and preformed interleukin-4 following immunological activation. Eur. J. Immunol. 26, 2493-2498 (1996).

117. Pelly, V. S. et al. IL-4-producing ILC2s are required for the differentiation of $\mathrm{TH} 2$ cells following Heligmosomoides polygyrus infection. Mucosal Immunol. 9, 1407-1417 (2016).

118. Okoye, I. S. \& Wilson, M. S. CD4+ Thelper 2 cellsmicrobial triggers, differentiation requirements and effector functions. Immunology 134, 368-377 (2011)

119. Cheever, A. W. et al. Anti-IL-4 treatment of Schistosoma mansoni-infected mice inhibits development of T cells and non-B, non-T cells expressing Th2 cytokines while decreasing egginduced hepatic fibrosis. J. Immunol. 153, 753-759 (1994).

120. Cheever, A. W., Finkelman, F. D. \& Cox, T. M. Anti-interleukin-4 treatment diminishes secretion of Th2 cytokines and inhibits hepatic fibrosis in murine schistosomiasis japonica. Parasite Immunol. 17 103-109 (1995).

121. Chiaramonte, M. G., Donaldson, D. D., Cheever, A. W. \& Wynn, T. A. An IL-13 inhibitor blocks the development of hepatic fibrosis during a T-helper type 2-dominated inflammatory response. J. Clin. Invest. 104, 777-785 (1999).

122. Mora, A. L. et al. Lung infection with $\gamma$-herpesvirus induces progressive pulmonary fibrosis in Th2-biase mice. Am. J. Physiol. Lung Cell. Mol. Physiol. 289 L711-L721 (2005).

123. Koth, L. L. et al. Aspergillus antigen induces robust Th2 cytokine production, inflammation, airway hyperreactivity and fibrosis in the absence of MCP-1 or CCR2. Respir. Res. 5, 12 (2004).

124. Westermann, W. Schobl, R., Rieber, E. P. \& Frank, K. H. Th2 cells as effectors in postirradiation pulmonary damage preceding fibrosis in the rat. Int. J. Radiat. Biol. 75, 629-638 (1999)

125. Braga, T. T. et al. MyD88 signaling pathway is involved in renal fibrosis by favoring a $\mathrm{TH} 2$ immune response and activating alternative $\mathrm{M} 2$ macrophages. Mol. Med. 18, 1231-1239 (2012).

126. Liu, L. et al. CD4 ${ }^{+}$T lymphocytes, especially Th2 cells, contribute to the progress of renal fibrosis. Am. J. Nephrol. 36. 386-396 (2012).

127. Avraham, T. et al. Th2 differentiation is necessary for soft tissue fibrosis and lymphatic dysfunction resulting from lymphedema. FASEB J. 27, 1114-1126 (2013)

128. Yoh, K., Ojima, M. \& Takahashi, S. Th2-biased GATA-3 transgenic mice developed severe experimental peritoneal fibrosis compared with Th1-biased T-bet and Th17-biased RORyt transgenic mice. Exp. Anim 64, 353-362 (2015).

129. Bobat, S et al. Soluble flagellin, FliC, induces an Ag-specific Th2 response, yet promotes T-betregulated Th 1 clearance of Salmonella typhimurium infection. Eur. J. Immunol. 41, 1606-1618 (2011).

130. Hollams, E. M et al. Th2-associated immunity to bacteria in teenagers and susceptibility to asthma. Eur. Respir. J. 36, 509-516 (2010)

131. Matzinger, P. The danger model: a renewed sense of self. Science 296, 301-305 (2002)

132. Guo, L. et al. Innate immunological function of TH2 cells in vivo. Nat. Immunol. 16, 1051-1059 (2015).

133. Endo, Y. et al. The interleukin-33-p38 kinase axis confers memory T helper 2 cell pathogenicity in the airway. Immunity 42, 294-308 (2015).

134. Van Dyken, S. J. et al. A tissue checkpoint regulates type 2 immunity. Nat. Immunol. 17, 1381-1387 (2016)

135. Chae, W. J. et al. The wnt antagonist dickkopf-1 promotes pathological type 2 cell-mediated inflammation. Immunity 44, 246-258 (2016). This study identified platelet-derived DKK1 as a novel inducer of $\mathrm{T}_{\mathrm{H}} 2$ cell polarization and type 2 cytokine expression.

136. Belperio, J. A. et al. The role of the Th2 CC chemokine ligand CCL17 in pulmonary fibrosis. J. Immunol. 173. 4692-4698 (2004)

37. Gordon, S. Alternative activation of macrophages Nat. Rev. Immunol. 3, 23-35 (2003).

138. Pesce, J. et al. The IL-21 receptor augments Th2 effector function and alternative macrophage activation. J. Clin. Invest. 116, 2044-2055 (2006). This paper showed that IL-21 signalling through $\mathrm{CD}^{+} \mathrm{T}$ cells is important for cytokine expression during type 2 disease and that IL-21 can augment and amplify IL-4- and IL-13-mediated activation of macrophages.
139. Coquet, J. M. et al. Interleukin-21-producing CD4+ T cells promote type 2 immunity to house dust mites. Immunity 43, 318-330 (2015)

This study demonstrated an important role for IL-21 in allergic asthma pathogenesis by potentiating $\mathrm{T}_{\mathrm{H}} 2$ cell functions and synergistically amplifying airway eosinophilia.

140. Barron, L. \& Wynn, T. A. Fibrosis is regulated by Th2 and Th 17 responses and by dynamic interactions between fibroblasts and macrophages. Am. J. Physiol. Gastrointest Liver Physiol. 300, G723-G728 (2011).

141. Sun, K., Tordjman, J., Clement, K. \& Scherer, P. E. Fibrosis and adipose tissue dysfunction. Cell Metab. 18, 470-477 (2013)

142. Yang, K. et al. T cell exit from quiescence and differentiation into Th2 cells depend on Raptor-mTORC1-mediated metabolic reprogramming. Immunity 39, 1043-1056 (2013).

143. Huang, S. C. et al. Cell-intrinsic lysosomal lipolysis is essential for alternative activation of macrophages. Nat. Immunol. 15, 846-855 (2014). This work found that fatty acid oxidation through lysosomal lipolysis is necessary for alternative activation of macrophages by IL-4 and IL- 13 .

144. Kannan, Y. et al. TPL-2 regulates macrophage lipid metabolism and M2 differentiation to control TH2-mediated immunopathology. PLoS Pathog. 12 e1005783 (2016).

145. Pesce, J. T. et al. Arginase-1-expressing macrophages suppress Th2 cytokine-driven inflammation and fibrosis. PLoS Pathog. 5, e1000371 (2009). This work demonstrated that arginase 1 expressio by macrophages is essential for regulating $\mathrm{T}$ cell responses by depleting amino acids necessary for T cell proliferation.

146. Nussbaum, J. C. et al. Type 2 innate lymphoid cells control eosinophil homeostasis. Nature 502 245-248 (2013).

This study identified ILC2s as a critical source of IL-5, maintaining eosinophil homeostasis and controlling circadian influx after eating.

147. Lee, M. W. et al. Activated type 2 innate lymphoid cells regulate beige fat biogenesis. Cell 160, 74-87 (2015).

This paper demonstrated that ILC2s control beige fat biogenesis through direct IL-13 signalling and recruitment of eosinophils, both of which promote the proliferation and differentiation of beige adipocyte precursors.

148. Brestoff, J. R. et al. Group 2 innate lymphoid cells promote beiging of white adipose tissue and limit obesity. Nature 519, 242-246 (2015).

149. Prahalad, P., Odegaard, J. I. \& Chawla, A. in The Th2 Type Immune Response in Health and Disease (eds Gause, W. C. \& Artis, D.) 155-169 (Springer Science + Business Media, 2016)

150. Pawlak, M., Lefebvre, P. \& Staels, B. Molecular mechanism of PPAR $\alpha$ action and its impact on lipid metabolism, inflammation and fibrosis in non-alcoholic fatty liver disease. J. Hepatol. 62, 720-733 (2015).

51. Mahajan, R., Lau, D. H. \& Sanders, P. Impact of obesity on cardiac metabolism, fibrosis, and function. Trends Cardiovasc. Med. 25, 119-126 (2015).

152. Bissell, D. M. Connective tissue metabolism and hepatic fibrosis: an overview. Semin. Liver Dis. 10 iii-iv (1990)

153. Eming, S. A., Wynn, T. A. \& Martin, P. Inflammation and metabolism in tissue repair and regeneration. Science 356, 1026-1030 (2017).

154. Yang, J. Q. et al. RhoA orchestrates glycolysis for TH2 cell differentiation and allergic airway inflammation. J. Allergy Clin. Immunol. 137, 231-245 (2016).

155. Pellicoro, A., Ramachandran, P., Iredale, J. P. \& Fallowfield, J. A. Liver fibrosis and repair: immune regulation of wound healing in a solid organ. Nat. Rev. Immunol. 14, 181-194 (2014)

156. Wynn, T. A. \& Barron, L. Macrophages: master regulators of inflammation and fibrosis. Semin. Liver Dis. 30, 245-257 (2010).

157. Borthwick, L. A. et al. Macrophages are critical to the maintenance of IL-13-dependent lung inflammation and fibrosis. Mucosal Immunol. 9, 38-55 (2016). This study showed that depletion of macrophages in the lung impairs the homing and effector response of $\mathrm{T}_{\mathrm{H}} 2$ cells, resulting in reduced fibrosis and inflammation.

158. Bronte, V., Serafini, P., Mazzoni, A., Segal, D. M. \& Zanovello, P. L-Arginine metabolism in myeloid cells controls T-lymphocyte functions. Trends Immunol. 24 302-306 (2003) 
159. Barron, L. et al. Role of arginase 1 from myeloid cells in th2-dominated lung inflammation. PLOS ONE 8 , e61961 (2013).

160. Satoh, T. et al. Identification of an atypical monocyte and committed progenitor involved in fibrosis. Nature 541, 96-101 (2017)

161. Vannella, K. M. et al. Incomplete deletion of IL-4R $\alpha$ by LysM ${ }^{\text {cre }}$ reveals distinct subsets of M2 macrophages controlling inflammation and fibrosis in chronic schistosomiasis. PLoS Pathog. 10, e 1004372 (2014).

This paper demonstrated that resident IL-4- and IL-13-activated macrophages are a crucial cell population necessary for controlling inflammation, whereas IL-4- and IL-13-activated recruited monocytes are important for controlling fibrosis during schistosomiasis.

162. Wynn, T. A. Fibrotic disease and the $T_{H} 1 / T_{H} 2$ paradigm. Nat. Rev. Immunol. 4, 583-594 (2004).

163. Iredale, J. P., Thompson, A. \& Henderson, N. C. Extracellular matrix degradation in liver fibrosis: biochemistry and regulation. Biochim. Biophys. Acta 1832, 876-883 (2013).

164. Madala, S. K. et al. Matrix metalloproteinase 12-deficiency augments extracellular matrix degrading metalloproteinases and attenuates IL-13-dependent fibrosis. J. Immunol. 184, 3955-3963 (2010).

165. Knipper, J. A. et al. Interleukin- 4 receptor $\alpha$ signaling in myeloid cells controls collagen fibril assembly in skin repair. Immunity 43, 803-816 (2015).

This study identified IL-4- and IL-13-activated macrophage-derived RELM $\alpha$ and subsequent induction of lysyl hydroxylase 2 as a critical mechanism of collagen fibril crosslinking.

166. Nair, M. G. et al. Alternatively activated macrophagederived RELM- $\alpha$ is a negative regulator of type 2 inflammation in the lung. J. Exp. Med. 206, 937-952 (2009).

167. Pesce, J. T. et al. Retnla (relma/fizz 1) suppresses helminth-induced Th2-type immunity. PLoS Pathog. 5 e1000393 (2009).

168. Bosurgi, L. et al. Macrophage function in tissue repair and remodeling requires IL-4 or IL-13 with apoptotic cells. Science 356, 1072-1076 (2017).

169. Minutti, C. M. et al. Local amplifiers of IL-4R $\alpha$-mediated macrophage activation promote repair in lung and liver. Science 356, 1076-1080 (2017).

170. de Kleer, I. M. et al. Perinatal activation of the interleukin-33 pathway promotes type 2 immunity in the developing lung. Immunity 45, 1285-1298 (2016).

171. Odegaard, J. I. et al. Perinatal licensing of thermogenesis by IL-33 and ST2. Cell 166, 841-854 (2016).

172. Rosenberg, H. F., Dyer, K. D. \& Foster, P. S Eosinophils: changing perspectives in health and disease. Nat. Rev. Immunol. 13, 9-22 (2013).

173. Minshall, E. M. et al. Eosinophil-associated TGF- $\beta 1$ mRNA expression and airways fibrosis in bronchial asthma. Am. J. Respiratory Cell. Mol. Biol. 17, 326-333 (1997)

174. Reiman, R. M. et al. Interleukin-5 (IL-5) augments the progression of liver fibrosis by regulating IL-13 activity. Infect. Immun. 74, 1471-1479 (2006).

175. Kopf, M. et al. IL-5-deficient mice have a developmental defect in CD5+ B-1 cells and lack eosinophilia but have normal antibody and cytotoxic T cell responses. Immunity 4, 15-24 (1996).

176. Swartz, J. M. et al. Schistosoma mansoni infection in eosinophil lineage-ablated mice. Blood 108 2420-2427 (2006).

177. Rothenberg, M. E. et al. Treatment of patients with the hypereosinophilic syndrome with mepolizumab. N. Engl. J. Med. 358, 1215-1228 (2008)

178. Chupp, G. L. et al. Efficacy of mepolizumab add-on therapy on health-related quality of life and markers of asthma control in severe eosinophilic asthma (MUSCA): a randomised, double-blind, placebocontrolled, parallel-group, multicentre, phase $3 \mathrm{~b}$ trial. Lancet Respir. Med. 5, 390-400 (2017).

179. Percopo, C. M. et al. Pulmonary eosinophils and their role in immunopathologic responses to formalininactivated pneumonia virus of mice. J. Immunol. 183 604-612 (2009)

180. Jacobsen, E. A. et al. Allergic pulmonary inflammation in mice is dependent on eosinophil-induced recruitment of effector T cells. J. Exp. Med. 205 699-710 (2008)
181. Lee, E. J. et al. Single high-dose irradiation aggravates eosinophil-mediated fibrosis through IL-33 secreted from impaired vessels in the skin compared to fractionated irradiation. Biochem. Bioph Res. Co 464 , 20-26 (2015).

182. Oyoshi, M. K. et al. Eosinophil-derived leukotriene C4 signals via type 2 cysteinyl leukotriene receptor to promote skin fibrosis in a mouse model of atopic dermatitis. Proc. Natl Acad. Sci. USA 109, 4992-4997 (2012)

183. Mesnil, C. et al. Lung-resident eosinophils represent a distinct regulatory eosinophil subset. J. Clin. Invest. 126, 3279-3295 (2016).

184. Hart, K. M. et al. Type 2 immunity is protective in metabolic disease but exacerbates NAFLD collaboratively with TGF- $\beta$. Sci. Transl. Med. 9 , eaal3694 (2017).

\section{This study demonstrated a previously} unappreciated link between hepatic eosinophils, type 2 cytokines and the severity of fibrosis in NAFLD.

185. Goh, Y. P. et al. Eosinophils secrete IL-4 to facilitate liver regeneration. Proc. Natl Acad. Sci. USA 110, 9914-9919 (2013)

186. Heredia, J. E. et al. Type 2 innate signals stimulate fibro/adipogenic progenitors to facilitate muscle regeneration. Cell 153, 376-388 (2013)

187. Nowarski, R., Jackson, R. \& Flavell, R. A. The stromal intervention: regulation of immunity and inflammation at the epithelial-mesenchymal barrier. Cell 168 362-375 (2017).

188. Gieseck, R. L. 3rd et al. Interleukin-13 activates distinct cellular pathways leading to ductular reaction, steatosis, and fibrosis. Immunity 45, 145-158 (2016).

This study demonstrated that ablating IL-13 signalling directly in fibroblasts eliminates fibrosis during several type 2-driven diseases and, similarly, that depleting IL-13 signalling in the hepatic epithelium impairs epithelial regeneration.

189. Wipff, P. J. \& Hinz, B. Integrins and the activation of latent transforming growth factor $\beta 1$ - an intimate relationship. Eur. J. Cell Biol. 87, 601-615 (2008)

190. Henderson, N. C. et al. Targeting of $\alpha_{v}$ integrin identifies a core molecular pathway that regulates fibrosis in several organs. Nat. Med. 19, 1617-1624 (2013).

191. Reed, N. I. et al. The $\alpha_{v} \beta_{1}$ integrin plays a critical in vivo role in tissue fibrosis. Sci. Transl. Med 7 288ra79 (2015).

192. Hartl, D. et al. Acidic mammalian chitinase is secreted via an ADAM17/epidermal growth factor receptor dependent pathway and stimulates chemokine production by pulmonary epithelial cells. J. Biol. Chem. 283, 33472-33482 (2008).

193. Zhu, Z. et al. Acidic mammalian chitinase in asthmatic Th2 inflammation and IL-13 pathway activation. Science 304, 1678-1682 (2004).

194. Lalaker, A., Nkrumah, L., Lee, W. K., Ramanathan, M. \& Lane, A. P. Chitin stimulates expression of acidic mammalian chitinase and eotaxin-3 by human sinonasal epithelial cells in vitro. Am. J. Rhinol. Allergy 23, 8-14 (2009).

195. Reese, T. A. et al. Chitin induces accumulation in tissue of innate immune cells associated with allergy. Nature 447, 92-96 (2007).

196. Van Dyken, S. J. et al. Chitin activates parallel immune modules that direct distinct inflammatory responses via innate lymphoid type 2 and $\gamma \delta$ T cells. Immunity 40, 414-424 (2014).

197. Fitz, L. J. et al. Acidic mammalian chitinase is not a critical target for allergic airway disease. $A m$. J. Respiratory Cell. Mol. Biol. 46, 71-79 (2012).

198. Vannella, K. M. et al. Acidic chitinase primes the protective immune response to gastrointestinal nematodes. Nat. Immunol. 17, 538-544 (2016). This study identified epithelial-derived AMCase as a crucial primer necessary for the full induction of type 2 immunity during infection with chitin-containing nematodes.

199. Van Dyken, S. J. et al. Spontaneous chitin accumulation in airways and age-related fibrotic lung disease. Cell 169, 497-509 (2017).

200. Denney, L. et al. Pulmonary epithelial cell-derived cytokine TGF- $\beta 1$ is a critical cofactor for enhanced innate lymphoid cell function. Immunity 43, 945-958 (2015)

This work identified a critical role for epithelial-derived TGF $\beta$ in recruiting pulmonary ILC2s, which subsequently drive allergic inflammation and hyperreactivity.
201. Gregory, L. G., Jones, C. P., Mathie, S. A., Pegorier, S. \& Lloyd, C. M. Endothelin-1 directs airway remodeling and hyper-reactivity in a murine asthma model. Allergy 68, 1579-1588 (2013).

202. Kuperman, D. A. et al. Direct effects of interleukin-13 on epithelial cells cause airway hyperreactivity and mucus overproduction in asthma. Nat. Med. 8 , 885-889 (2002)

203. Mahapatro, M. et al. Programming of intestinal epithelial differentiation by IL-33 derived from pericryptal fibroblasts in response to systemic infection. Cell Rep. 15, 1743-1756 (2016).

204. Finkelman, F. D. et al. Effects of interleukin 12 on immune responses and host protection in mice infected with intestinal nematode parasites. J. Exp. Med. 179, 1563-1572 (1994).

205. Szabo, S. J., Jacobson, N. G., Dighe, A. S., Gubler, U. \& Murphy, K. M. Developmental commitment to the Th2 lineage by extinction of IL-12 signaling. Immunity 2, 665-675 (1995)

206. Else, K. J., Finkelman, F. D., Maliszewski, C. R. \& Grencis, R. K. Cytokine-mediated regulation of chronic intestinal helminth infection. J. Exp. Med. 179, 347-351 (1994)

207. Krausgruber, T. et al. IRF5 promotes inflammatory macrophage polarization and $\mathrm{TH} 1-\mathrm{TH} 17$ responses. Nat. Immunol. 12, 231-238 (2011).

208. Fiorentino, D. F. et al. IL-10 acts on the antigen presenting cell to inhibit cytokine production by $T h 1$ cells. J. Immunol. 146, 3444-3451 (1991).

209. Del Prete, G. et al. Human IL-10 is produced by both type 1 helper (Th1) and type 2 helper (Th2) T cell clones and inhibits their antigen-specific proliferation and cytokine production. J. Immunol. 150, 353-360 (1993).

210. Zheng, T. et al. IL-13 receptor $\alpha 2$ selectively inhibits $\mathrm{IL}-13$-induced responses in the murine lung. J. Immunol. 180, 522-529 (2008)

211. Mentink-Kane, M. M. et al. IL-13 receptor $\alpha 2$ downmodulates granulomatous inflammation and prolongs host survival in schistosomiasis. Proc. Natl Acad. Sci. USA 101, 586-590 (2004).

212. Mentink-Kane, M. M. et al. Accelerated and progressive and lethal liver fibrosis in mice that lack interleukin (IL)-10, IL-1 2p 40, and IL-13R $\alpha 2$. Gastroenterology 141, 2200-2209 (2011).

213. Badalyan, V. et al. TNF- $\alpha /$ /L- 17 synergy inhibits IL- 13 bioactivity via IL-13R $\alpha 2$ induction. J. Allergy Clin. Immunol. 134, 975-978 (2014).

214. He, C. H. et al. Chitinase 3 -like 1 regulates cellular and tissue responses via IL-13 receptor $\alpha 2$. Cell Rep. 4 , 830-841 (2013)

215. Lee, C. M. et al. IL-13R $\alpha 2$ uses TMEM219 in chitinase 3 -like-1-induced signalling and effector responses. Nature Commun. 7, 12752 (2016)

216. Plataki, M. et al. Expression of apoptotic and antiapoptotic markers in epithelial cells in idiopathic pulmonary fibrosis. Chest 127, 266-274 (2005).

217. Schafer, M. J. et al. Cellular senescence mediates fibrotic pulmonary disease. Nat. Commun. 8, 14532 (2017).

218. Munoz-Espin, D. \& Serrano, M. Cellular senescence: from physiology to pathology. Nat. Rev. Mol. Cell Biol. $15,482-496(2014)$

219. Hagimoto, N. et al. Induction of apoptosis and pulmonary fibrosis in mice in response to ligation of Fas antigen. Am. J. Respiratory Cell. Mol. Biol. 17. 272-278 (1997)

220. Hagimoto, N., Kuwano, K., Nomoto, Y., Kunitake, R. \& Hara, N. Apoptosis and expression of Fas/Fas ligand mRNA in bleomycin-induced pulmonary fibrosis in mice. Am. J. Respiratory Cell. Mol. Biol. 16, 91-101 (1997).

221. Ramos, C. et al. Fibroblasts from idiopathic pulmonary fibrosis and normal lungs differ in growth rate, apoptosis, and tissue inhibitor of metalloproteinases expression. Am. J. Respiratory Cell. Mol. Biol. 24, 591-598 (2001)

222. Uhal, B. D. et al. Alveolar epithelial cell death adjacent to underlying myofibroblasts in advanced fibrotic human lung. Am. J. Physiol. 275, L1192-L1199 (1998).

223. Laplante, P. et al. Caspase-3-mediated secretion of connective tissue growth factor by apoptotic endothelial cells promotes fibrosis. Cell Death Differ 17, 291-303 (2010)

224. Wang, L. et al. Induction of secondary apoptosis, inflammation, and lung fibrosis after intratracheal instillation of apoptotic cells in rats. Am. J. Physiol. Lung Cell. Mol. Physiol. 290, L695-L702 (2006). 
225. Desmouliere, A., Redard, M., Darby, I. \& Gabbiani, G. Apoptosis mediates the decrease in cellularity during the transition between granulation tissue and scar. Am. J. Pathol. 146, 56-66 (1995).

226. Murphy, F. R. et al. Inhibition of apoptosis of activated hepatic stellate cells by tissue inhibitor of metalloproteinase- 1 is mediated via effects on matrix metalloproteinase inhibition: implications for reversibility of liver fibrosis. J. Biol. Chem. 277 11069-11076 (2002).

227. Lee, C. S. et al. Boosting apoptotic cell clearance by colonic epithelial cells attenuates inflammation in vivo. Immunity 44, 807-820 (2016).

228. Han, C. Z. et al. Macrophages redirect phagocytosis by non-professional phagocytes and influence inflammation. Nature 539, 570-574 (2016).

229. Cummings, R. J. et al. Different tissue phagocytes sample apoptotic cells to direct distinct homeostasis programs. Nature 539, 565-569 (2016).

230. Ramalingam, T. R. et al. Enhanced protection from fibrosis and inflammation in the combined absence of IL-13 and IFN- $\gamma$. J. Pathol. 239, 344-354 (2016). This study demonstrated a compensatory increase in IFN $\gamma$ during IL-13 blockade, which increased type 1 cytotoxicity and necrosis, and additionally demonstrated that dual blockade of IL-13 and IFN $\gamma$ resulted in further reductions in fibrosis and elimination of rebound type 1 inflammation and toxicity.

231. Choy, D. F. et al. $T_{H} 2$ and $T_{H} 17$ inflammatory pathways are reciprocally regulated in asthma. Sci. Transl. Med. 7, 301 ra129 (2015)

This study identified distinct $T_{H} 2$ and $T_{H} 17$ cell-driven asthma subsets, demonstrated that blockade of IL-13 or IL-17 in the respective subsets resulted in a compensatory increase in $\mathrm{T}_{\mathrm{H}} 17$ - or $\mathrm{T}_{\mathrm{H}}$ 2-driven inflammation and showed that dual blockade of IL-13 and IL-17 resulted in maximal therapeutic efficacy.

Acknowledgements

This work was supported by the Intramural Research Program of the US National Institutes of Health National Institute of Allergy and Infectious Diseases.

\section{Competing interests statement}

The authors declare no competing interests.

\section{Publisher's note}

Springer Nature remains neutral with regard to jurisdictional claims in published maps and institutional affiliations.

\section{ONLINE CORRESPONDENCE $\$}

Nature Reviews Immunology publishes items of correspondence online. Such contributions are published at the discretion of the Editors and can be subject to peer review. Correspondence should be no longer than 500 words with up to 15 references and should represent a scholarly attempt to comment on a specific Review or Perspective article that has been published in the journal. To view correspondence, please go to our homepage at: http://www.nature.com/nri and follow the link from the current table of contents. To cite correspondence, please use its doi number.

The following correspondence has recently been published:

Science not art: statistically sound methods for identifying subsets in multi-dimensional flow and mass cytometry data sets

Darya Y. Orlova, Leonore A. Herzenberg and Guenther Walther

doi: 10.1038/nri.2017.150

REPLY Response to Orlova et al. "Science not art: statistically sound methods for identifying subsets in multi-dimensional flow and mass cytometry data sets"

Yvan Saeys, Sofie Van Gassen and Bart Lambrecht

doi: 10.1038/nri.2017.151

This correspondence relates to the article:

Computational flow cytometry: helping to make sense of high-dimensional immunology data.

Yvan Saeys, Sofie Van Gassen and Bart Lambrecht

Nat. Rev. Immunol. 16, 449-462 2016 